ARTICLE

\title{
Distinct subtypes of proprioceptive dorsal root ganglion neurons regulate adaptive proprioception in mice
}

Haohao Wu (1) 1, Charles Petitpré1,8, Paula Fontanet (10 1,8, Anil Sharma (1) 1, Carmelo Bellardita 1,2, Rolen M. Quadros ${ }^{3}$, Paulo R. Jannig ${ }^{4}$, Yiqiao Wang ${ }^{1}$, J. Alexander Heimel ${ }^{5}{ }^{5}$, Kylie K. Y. Cheung ${ }^{1}$, Simone Wanderoy', Yang Xuan', Konstantinos Meletis (1) 1, Jorge Ruas (10) ${ }^{4}$, Channabasavaiah B. Gurumurthy (D) ${ }^{3,6}$, Ole Kiehn ${ }^{1,2}$, Saida Hadjab (D) ${ }^{1}$ \& François Lallemend (1) 1,7凶

Proprioceptive neurons (PNs) are essential for the proper execution of all our movements by providing muscle sensory feedback to the central motor network. Here, using deep single cell RNAseq of adult PNs coupled with virus and genetic tracings, we molecularly identify three main types of PNs ( $\mathrm{la}, \mathrm{lb}$ and II) and find that they segregate into eight distinct subgroups. Our data unveil a highly sophisticated organization of PNs into discrete sensory input channels with distinct spatial distribution, innervation patterns and molecular profiles. Altogether, these features contribute to finely regulate proprioception during complex motor behavior. Moreover, while Ib- and II-PN subtypes are specified around birth, la-PN subtypes diversify later in life along with increased motor activity. We also show la-PNs plasticity following exercise training, suggesting la-PNs are important players in adaptive proprioceptive function in adult mice.

\footnotetext{
${ }^{1}$ Department of Neuroscience, Karolinska Institutet, Stockholm, Sweden. ${ }^{2}$ Department of Neuroscience, University of Copenhagen, Copenhagen, Denmark.

${ }^{3}$ Mouse Genome Engineering Core Facility, Department of Pharmacology and Experimental Neuroscience, College of Medicine, University of Nebraska Medical Center, Omaha, NE, USA. ${ }^{4}$ Department of Physiology and Pharmacology, Karolinska Institutet, Stockholm, Sweden. ${ }^{5}$ Cortical Structure \& Function Group, Netherlands Institute for Neuroscience, Amsterdam, The Netherlands. ${ }^{6}$ Department of Pharmacology and Experimental Neuroscience, College of Medicine, University of Nebraska Medical Center, Omaha, NE, USA. ${ }^{7}$ Ming Wai Lau Centre for Reparative Medicine, Stockholm Node, Karolinska Institutet, Stockholm, Sweden. ${ }^{8}$ These authors contributed equally: Charles Petitpré, Paula Fontanet. ${ }^{\circledR}$ email: francois.lallemend@ki.se
} 
$\mathrm{P}$ roprioceptive feedback constantly monitors consequences of motor action. It is essential for the efficiency, precision, and robustness of movements in all motile animals and necessary for locomotor recovery in pathological conditions ${ }^{1,2}$. In mammals, the gatekeepers of the proprioceptive system are the proprioceptive neurons (PNs), residing in the dorsal root ganglia (DRG) along the spinal cord, projecting peripherally to skeletal muscles and continuously reporting to the central nervous sys$\mathrm{tem}^{3}$. Three functional types of PNs have been identified from anatomical and physiological studies: the stretch-sensitive Ia- and II-PNs, which innervate muscle spindles (MSs), and the forcesensitive Ib-PNs, which innervate Golgi tendon organs (GTOs) (Fig. 1a $)^{3-5}$. While this classic view on the hard-wired proprioceptive system has not been challenged for decades, the understanding of the central motor circuits has evolved ${ }^{6,7}$, revealing its far more sophisticated organization to endow the rich repertoire of behaviors and high-level of plasticity to accommodate the everchanging activities in mammals. It is thus unclear whether a larger diversity and plasticity of cell type and circuit is a basis for governing proprioceptive functions for accurate motor control.

Recent efforts in transcriptomic profiling of DRG neurons have generated a molecular catalog of somatosensory neurons and start to shed light on the transcriptional heterogeneity within some sensory types, e.g., nociceptors and mechanoreceptors ${ }^{8-11}$. Results from these studies demonstrate the existence of a dozen subtypes of nociceptive neurons, three subtypes of skin mechanoreceptors, and one homogenous population of PNs. Therefore, the understanding of the molecular diversity within PNs remains limited. This likely reflects the natural challenge of molecular profiling of PNs, i.e., its low abundance among DRG neurons and the transcriptomic similarity between the subtypes. Here, combining deep scRNAseq of large number of PNs, mouse genetics, anatomical tracing, and exercise training, we report a highly specialized organization of the proprioceptive system comprising eight subtypes with distinct molecular profiles, spatial distribution and patterns of connectivity, revealing high levels of proprioceptive sensory inputs specialization. Moreover, their versatile composition in adult after sustained exercise training suggests plasticity with impact for sensory performance adaptability.

\section{Results}

Deep single-cell RNAseq reveals molecular diversity of adult PNs. To obtain an enriched population of PNs amongst DRG neurons, we FAC sorted cells expressing tdTomato (TOM) from C5 to T1 DRG of postnatal stage 54 (P54) $P V^{C r e}$;Ai14 mice and processed them with Smart-seq2 protocol (Fig. 1b, $c$ and Supplementary Fig. 1a) ${ }^{12}$. A total of 1109 PNs $\left(\mathrm{Whrn}^{+}, \mathrm{Pvalb}^{+}\right.$, Run $\times 3^{+}, \mathrm{Ntrk}^{+}$and $E t v 1^{+}$) passed quality control with a high gene coverage $(\sim 11,000$ genes detected per cell) (Supplementary Fig. 1b, c), allowing deep analysis of their molecular profiles. A small number of mechanoreceptors $\left(\mathrm{Ntrk}^{+}\right.$or $\mathrm{Ret}^{+}, \mathrm{Pvalb}^{\text {low }}$ and $\left(\mathrm{Whr}^{-}\right)$were also identified and thus excluded from the subsequent analysis (Supplementary Fig. 1d). Transcriptomic analysis $^{13}$ (see "Methods" for details) revealed highly diversified picture of adult PNs, comprising eight molecularly distinct clusters (Fig. 1d), each with numerous genetic markers (Fig. 1e and a

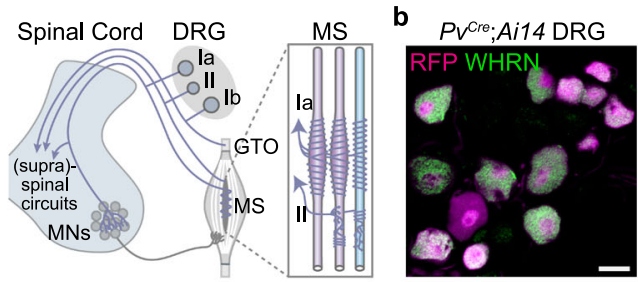

C DRG dissection Dissociation Cell capture Sequencing

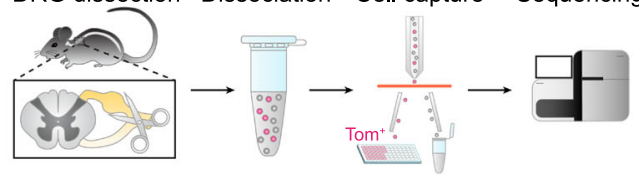

f In vivo validation of all clusters
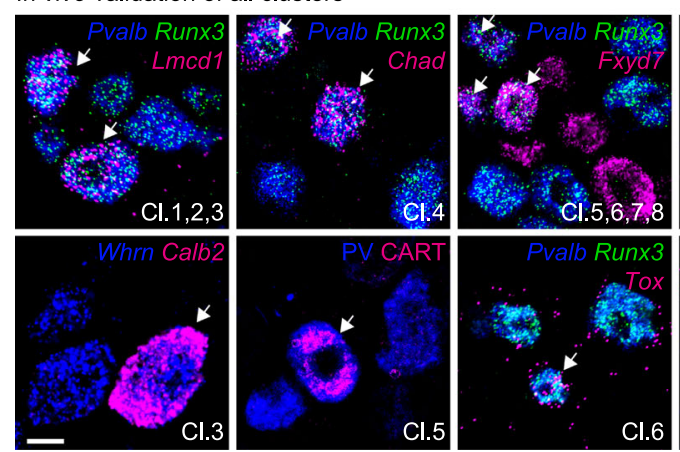
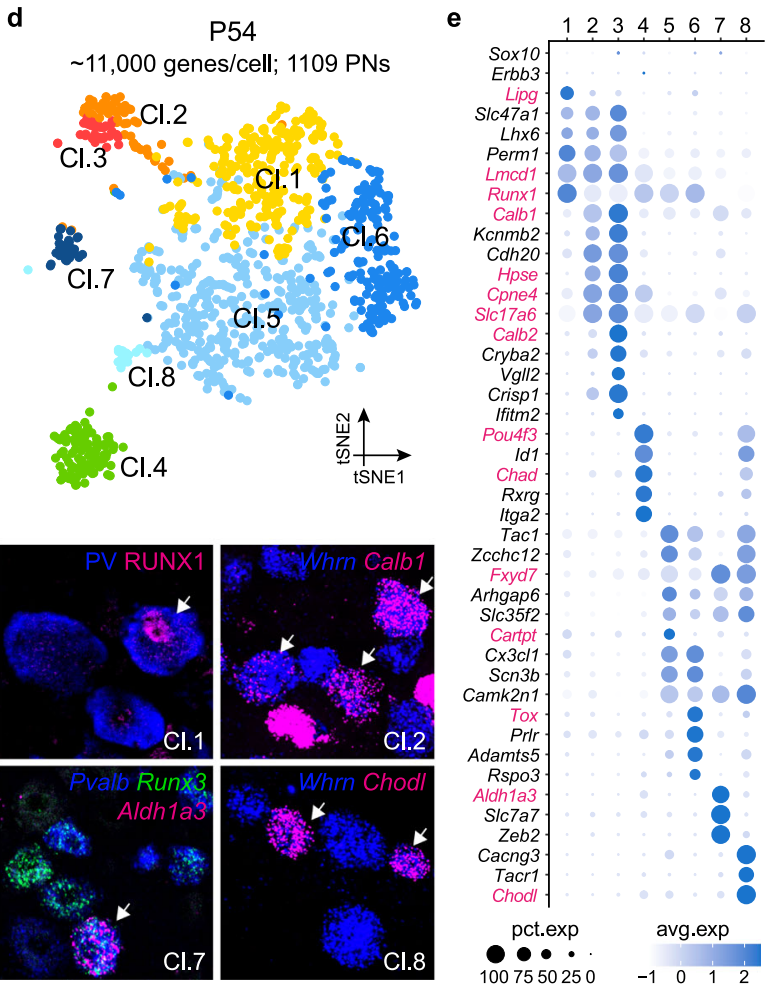

Fig. 1 Deep scRNAseq reveals large heterogeneity among adult PNs. a Schematic depiction of the central and peripheral innervation patterns of the three major PN types in the adult. b Brachial DRG section of P40 PVCre;Ai14 mouse stained for PN marker WHRN. Scale bar: $20 \mu$ m. c Schematic illustration of the workflow for scRNAseq of P54 PNs. d Adult PNs single-cell transcriptomes visualized with t-distributed stochastic neighbor embedding (tSNE), color-

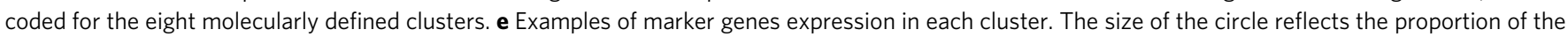
cells expressing the marker gene in a cluster, and the color intensity reflects its average expression level within that cluster. The markers used in subsequent analysis are indicated in magenta. $\mathbf{f}$ In vivo validation of the clusters of PNs by RNAscope and immunohistochemistry using the identified markers (magenta) on P54 brachial DRG sections. PNs are labeled by PV for immunohistochemistry, and Pvalb/Runx3 or Whrn for RNAscope. Scale bar: $20 \mu \mathrm{m}$. 

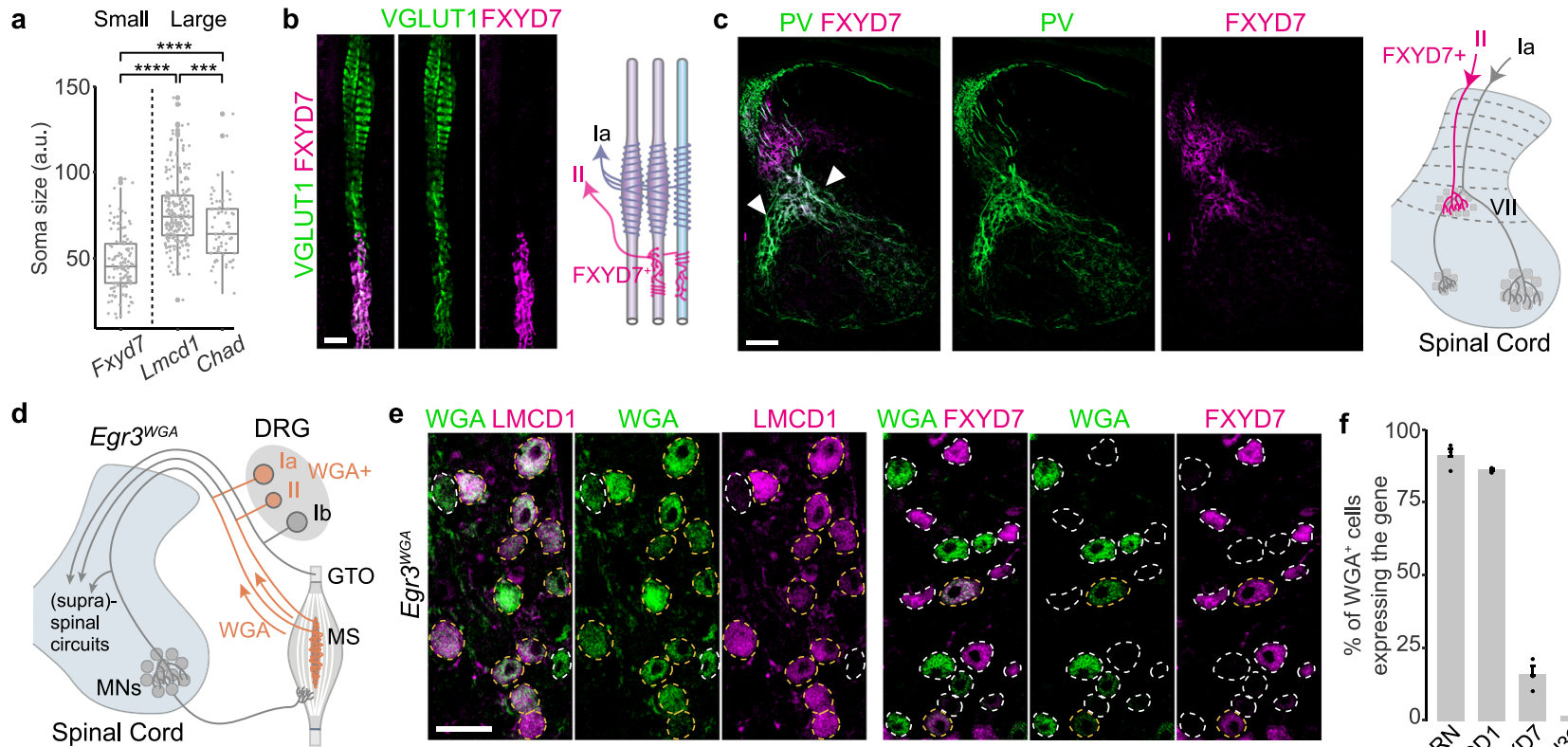

e WGA LMCD1 WGA
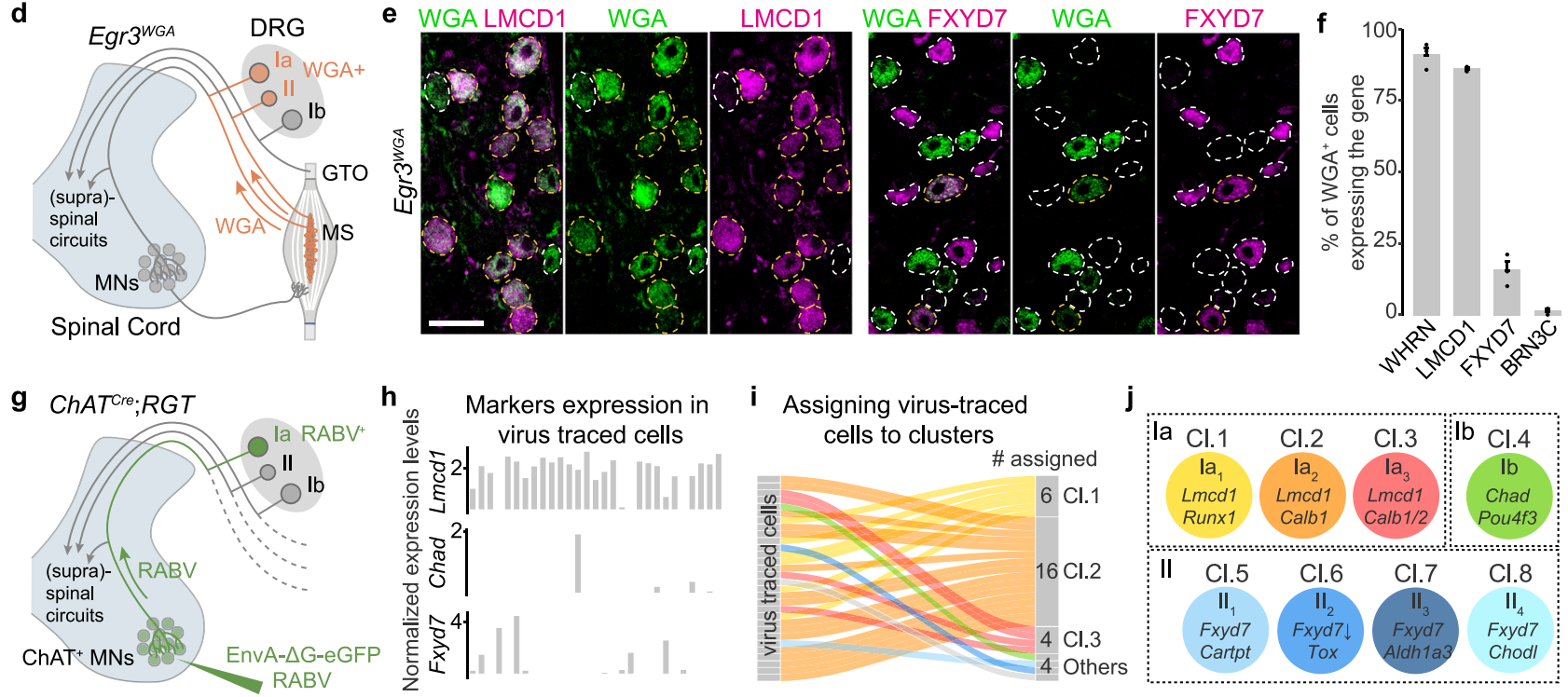

Fig. 2 Identification of the major PN types. a Quantification of cross-nucleus soma sizes of the three major PN populations depicted in box and whisker plot. Lower and upper hinges: first and third quartiles; the horizontal line: median; the whiskers extend to the value no further than 1.5 * IQR from the hinge; large dots: outliers. Each dot represents a cell: Fxyd7 + PNs ( $n=124$ cells), $L m c d 1^{+}$PNs $\left(n=227\right.$ cells), Chad + PNs $\left(n=69\right.$ cells). Two-tailed $t$-test, ${ }^{\star \star \star \star} p<$ $0.0001,{ }^{\star \star \star} p<0.001$. b Longitudinal section of muscle spindle (MS) stained for VGLUT1 and FXYD7. Scale bar: $20 \mu \mathrm{m}$. Right panel: schematic depiction of the finding. c Transverse section of P4 spinal cord stained for PV and FXYD7. Scale bar: $100 \mu \mathrm{m}$. Right panel: schematic depiction of the finding. d Genetic strategy to label MS-innervating PNs in Egr3WGA mice. e Representative image showing co-labeling of DRG neurons by WGA and LMCD1 or FXYD7 in DRG sections from P14 Egr3WGA mice. Scale bar: $50 \mu \mathrm{m}$. f Quantification of WGA+ cells expressing WHRN, LMCD1, FXYD7, and BRN3C, respectively, in P14 brachial DRG ( $n=3$ animals). Data are presented as mean \pm SEM; dots represent values from individual animals. $\mathbf{g}$ Trans-monosynaptic rabies virus tracing strategy to label la-PNs in adult ChATCre;RGT mice. $\mathbf{h}$ Bar plots showing that the majority of the rabies virus infected PNs express $L m c d 1$ but not Fxyd7 or Chad. i Assignment of virus infected PNs to the identified eight PN clusters based on their overall transcriptomic similarity. $\mathbf{j}$ A summary of correspondence between the known functional types of PNs and the cell clusters identified by scRNAseq. Source data are provided as a Source Data file.

Supplementary Fig. 1e). Among those, we observed transcriptomic similarities within clusters (cl.) 1-3 $\left(\operatorname{Lmcd1} 1^{+}\right)$and within cl.5-8 $\left(\mathrm{Fxyd}^{+}\right)$, which together with the cl.4 $\left(\mathrm{Chad}^{+}\right)$ form three major groups (see "Methods" for details). We further validated that all eight clusters and three major groups exist in vivo using the newly identified markers (Fig. 1f).

Molecular identification of Ia-, Ib-, and II-PNs. To identify the main functional types of PNs (Ia, Ib, and II) among the molecularly distinct clusters, we first analyzed the three major groups of PNs as defined by the expression of the new marker genes Lmcd1 (cl.1-3), Chad (cl.4), and Fxyd7 (cl.5-8), which together cover all PNs at brachial level. Combinatorial expression analysis of the three markers showed minimal overlapping between Fxyd7 and Lmcd1 or Fxyd7 and Chad, and few Chad ${ }^{+}$PNs expressing Lmcd1 but systematically at very low levels (Supplementary Fig. 2a, b). Morphologically, PN types can be distinguished by their axon diameter (medium: II-PNs: large: Ia- and Ib-PNs), which is proportional to their soma size and correlates with their conduction velocities ${ }^{14,15}$. We found that Fxyd7 ${ }^{+}$PNs exhibited on average smaller soma size compared to $\mathrm{Lmcd1}^{+}$and $\mathrm{Chad}^{+}$ PNs (Fig. 2a and Supplementary Fig. 2c). In addition, FXYD7+ PNs peripherally innervated MSs with endings invariably exhibiting "flower-spray"-like structure and positioning away from the central part of the MSs (Fig. 2c) ) $^{3,16}$ and centrally terminated at lamina VII of the spinal cord (Fig. 2c) ${ }^{17,18}$. These distinct attributes strongly suggest that FXYD7 ${ }^{+}$PNs represent II-PNs.

To assign the two populations of large size PNs marked by the expression of $L m c d 1$ or Chad to either Ia-PNs (innervating MSs) or Ib-PNs (innervating GTOs), we used the $E g r 3^{W G A}$ mouse line in which wheat germ agglutinin (WGA) is expressed by the Egr3 ${ }^{+}$ intrafusal muscle fibers of MSs and back-traces Ia- and II- but not Ib-PNs in DRG (Fig. 2d) ${ }^{19}$. In this mouse line, the selective expression of WGA in the central part of MSs would preferentially trace afferents of Ia-PNs (Supplementary Fig. 2d). We found that $91 \%$ of $\mathrm{WGA}^{+}$cells were PNs, out of which the majority were $\mathrm{LMCD}^{+}$(Fig. 2e, f) and $83 \%$ of $\mathrm{LMCD}^{+} \mathrm{PNs}$ were $\mathrm{WGA}^{+}$. In contrast, WGA was virtually absent from $\mathrm{BRN} \mathrm{C}^{+}$PNs (BRN3C, coded by Pou4f3, marks the Chad ${ }^{+} \mathrm{cl} .4$, while we could not obtain a working CHAD antibody) (Fig. 2f), indicating that they represent Ib-PNs. We also observed $15 \%$ of 
$\mathrm{WGA}^{+}$cells expressing FXYD7 (Fig. 2e, f), further supporting the type II identity of FXYD7 ${ }^{+}$PNs. These results, together with the larger soma size of $L m c d 1^{+}$PNs (Fig. 2a), strongly suggest that LMCD1 ${ }^{+}$PNs are Ia-PNs. To confirm this, we set to obtain IaPNs transcriptome specifically using a rabies virus-mediated trans-synaptic tracing strategy to selectively label Ia-PNs following motor neurons infection in ChAT ${ }^{\mathrm{Cre}} ; R G T$ mice (Fig. $\left.2 \mathrm{~g}\right)^{20}$. Five days post infection, we performed scRNAseq on the isolated $\mathrm{eGFP}^{+}$neurons from the virus-infected DRG, showing that most of the virus-infected PNs (most, if not all, are Ia-PNs) expressed Lmcd1, but not Fxyd7 or Chad (Fig. 2h) and 26 out of 30 virusinfected PNs were unbiasedly assigned to cl.1-3 using machine learning algorithm (Fig. $2 \mathrm{i})^{21}$, further confirming the type Ia identity of the $\mathrm{LMCD1}^{+}$PNs.

Altogether, we have identified the unique genetic signatures of the three major types of PNs (Ia, Ib, and II) and provided a molecular framework for PNs classification (Fig. 2j and Supplementary Dataset 1). Our scRNAseq analysis also reveals a large diversity of Ia- and II-PNs, implying a sophisticated organization of proprioceptive feedback from MSs.

\section{Functional subdivisions of PNs}

Main PN types. The discovery of marker genes for the main types of PNs enables us to answer how PN types and their associated sensory feedback are distributed to different skeletal muscles that have distinct functions and performance requirements. We found that the proportion of the Ia-PNs and II-PNs was relatively constant across all spinal segments analyzed (Fig. 3a, b and Supplementary Fig. 3a). However, at the level of individual muscles, the composition of Ia- and II-PNs nerve endings within MSs varied dramatically: most MSs in biceps were either intermediate or complex (with one or two II-PN endings in addition to one Ia-PN ending), whereas MSs in triceps were mostly simple, i.e., devoid of II-PNs innervation (Supplementary Fig. 3b). This suggests that the type of MS feedback (velocity vs. length of stretch) transmitted to the central motor circuits varies between muscles.

While Ia- and II-PNs were found at all levels of the rostrocaudal axis, Ib-PNs, which innervate GTOs, were almost exclusive to the brachial and lumbar DRG that supply limb muscles in addition to axial muscles (Fig. 3b). In support of this, we did not observe any GTOs in the mouse intercostal and erector spinae muscles (thoracic axial muscles) using VGLUT1 staining. This suggests that axial muscles, in large part, lack GTOs and associated sensory feedback.

Altogether, these data indicate that Ia, Ib and II sensory afferents are allocated differently to muscles, suggesting distinct sensory feedback demands from the central nervous system for distinct muscles.

$P N$ subtypes. We next set to understand the subdivisions within the main types of PNs through morphological and anatomical characterization, in order to address the functional relevance of the newly identified subtypes. Among Ia-PN subtypes, we observed that $\mathrm{Ia}_{1}-\mathrm{PNs}$ exhibited relatively smaller soma sizes and higher abundance in all DRG analyzed (Fig. $3 c$, d), while $\mathrm{Ia}_{2}$ - and $\mathrm{Ia}_{3}-\mathrm{PNs}$ exhibited larger soma sizes and could only be found in the DRG of brachial and lumbar segments innervating the limbs (Fig. 3c, d and Supplementary Fig. 3c, d). In line with this, using Calb1 ${ }^{d g C r e}$;Ail4 mice, the spinal $\mathrm{VGLUT1}^{+} / \mathrm{RFP}^{+}$terminal boutons of $\mathrm{Ia}_{2}-$ and $\mathrm{Ia}_{3}-\mathrm{PNs}$ were systematically found in the lateral motor column (LMC) area (Fig. 3e), which is occupied by limb-innervating MNs. The analysis of gene expression profile of $\mathrm{Ia}_{2}-$ and $\mathrm{Ia}_{3}-\mathrm{PNs}$ highlighted the genes sets related to synaptic proteins, ion channels, metabolic process and neurofilament
(Supplementary Fig. 3e), which together with their morphological and anatomical profile, suggest that they represent specific limbinnervating Ia-PN subtypes with higher activity demand and faster conduction velocity. Interestingly, $\mathrm{Ia}_{3}$-PNs were specifically enriched in C6 at brachial level (Fig. 3d), suggesting that they innervate selective muscle targets. In support of this, using $\mathrm{Calb2}_{2}{ }^{\mathrm{Cre}} ; \mathrm{Ai14}$, we found that in the forelimb, nerve endings of $\mathrm{Ia}_{3}-\mathrm{PNs}$ were much more abundant in MSs of the dorsal distal muscles of the limb, which are principal extensors of the wrist and digits (Fig. 3f, g). More specifically, $\sim 80 \%$ of MSs in ECR were innervated by $\mathrm{Ia}_{3}$-PNs, while MSs in triceps were completely devoid of $\mathrm{Ia}_{3}-\mathrm{PNs}$ innervation (Fig. 3g). Moreover, while Ia-PNs are known to innervate both nuclear bag and chain fibers, we observed that $\mathrm{Ia}_{3}-\mathrm{PNs}$ specifically spiraled around the nuclear bag fibers (Fig. 3h), which are more sensitive to the dynamic changes of muscles. In comparison with $\mathrm{Ia}_{2}-\mathrm{PNs}, \mathrm{Ia}_{3}-\mathrm{PNs}$ showed a particularly high expression of genes associated with energy metabolism, suggesting an even higher energy demand for this subtype (Supplementary Fig. 3f). Hence, these data suggest that $\mathrm{Ia}_{3}-\mathrm{PNs}$ represent a muscle-specific Ia-PN subtype with higher dynamic intrinsic properties and muscle-specific contribution to MSs sensory feedback.

Although all II-PN subpopulations exhibited generally smaller soma size compared with Ia- and Ib-PNs, the $\mathrm{II}_{3}-\mathrm{PNs}$ were on average larger (Fig. 3i), suggesting that they might have the fastest conduction velocity among II-PNs. Anatomically, $\mathrm{II}_{1^{-}}, \mathrm{II}_{3^{-}}$and $\mathrm{II}_{4}-\mathrm{PNs}$ were all found in brachial and lumbar DRG that innervate mostly the limbs and some axial muscles (Fig. 3j). In contrast, $\mathrm{II}_{2}$ - $\mathrm{PNs}$ were proportionally more abundant in the thoracic DRG that supply only axial muscles (Fig. $3 \mathrm{j}$ ). We found that $46.8 \pm 11.9 \%$ and only $11.4 \pm 3.6 \%$ of $\mathrm{II}_{2}$-PNs were Fxyd $7^{+}$in brachial and thoracic DRG, respectively, suggesting that $\mathrm{II}_{2}-\mathrm{PNs}$ might specifically innervate axial and intercostal muscles ${ }^{22}$.

Altogether, these data extended our current view on the modular organization of proprioceptive feedback and revealed 8 functional subtypes of PNs with distinguishable molecular profiles, spatial distributions (Fig. 3k), and connectivity patterns, suggesting another level of organization logic of the proprioceptive feedback.

Molecular signatures of neuronal communication pattern in PN subtypes. In addition to the anatomical distribution and connectivity, the transmission of the muscle proprioceptive feedback to the central motor circuits relies on distinct operational components that define their neurotransmission mode and functionality. Using differential expression analysis, we have identified numerous genes that were specifically expressed or enriched in each PN subtype (Supplementary Dataset 1). We further categorized those genes according to their functions and, while further analysis will be necessary to confirm their expression in situ, this list of candidate genes could serve as a basis for understanding the different properties of PN subtypes. Many genes for general cellular functions, including cytoskeleton, adhesion molecules, transcription factors, cell-to-cell signaling, and neuropeptides/hormones and receptors were differentially expressed amongst PNs, and are presented in Supplementary Fig. 4a-e. We next analyzed expression of genes, which belong to functional categories that characterize the physiological properties of mechanosensory neurons, including mechanosensitive ion channels (MSICs), integrin-mediated mechanotransduction, voltage-gated ion channels (VGICs) and neurotransmitter transport and receptors (Fig. 4a-f). While many MSICs, including Piezo $^{23}$, were found equally expressed in all PN subtypes (Fig. 4b), we identified six MSICs that were differentially expressed among them, indicating that each subtype of PNs 


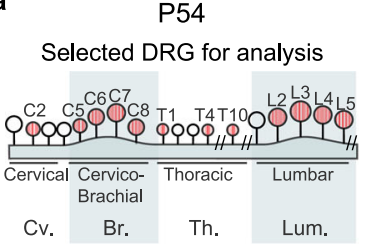

b Spatial distribution of 3 major types of PNs

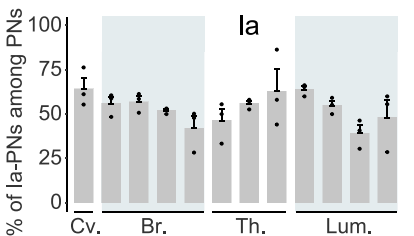

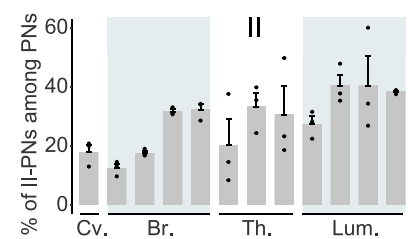

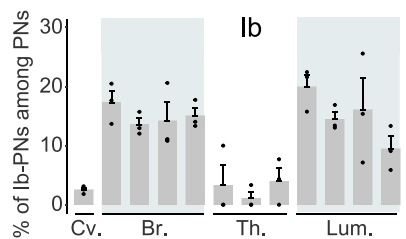

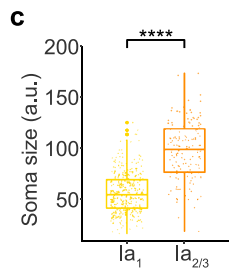

d Spatial distribution of subtypes of la-PNs
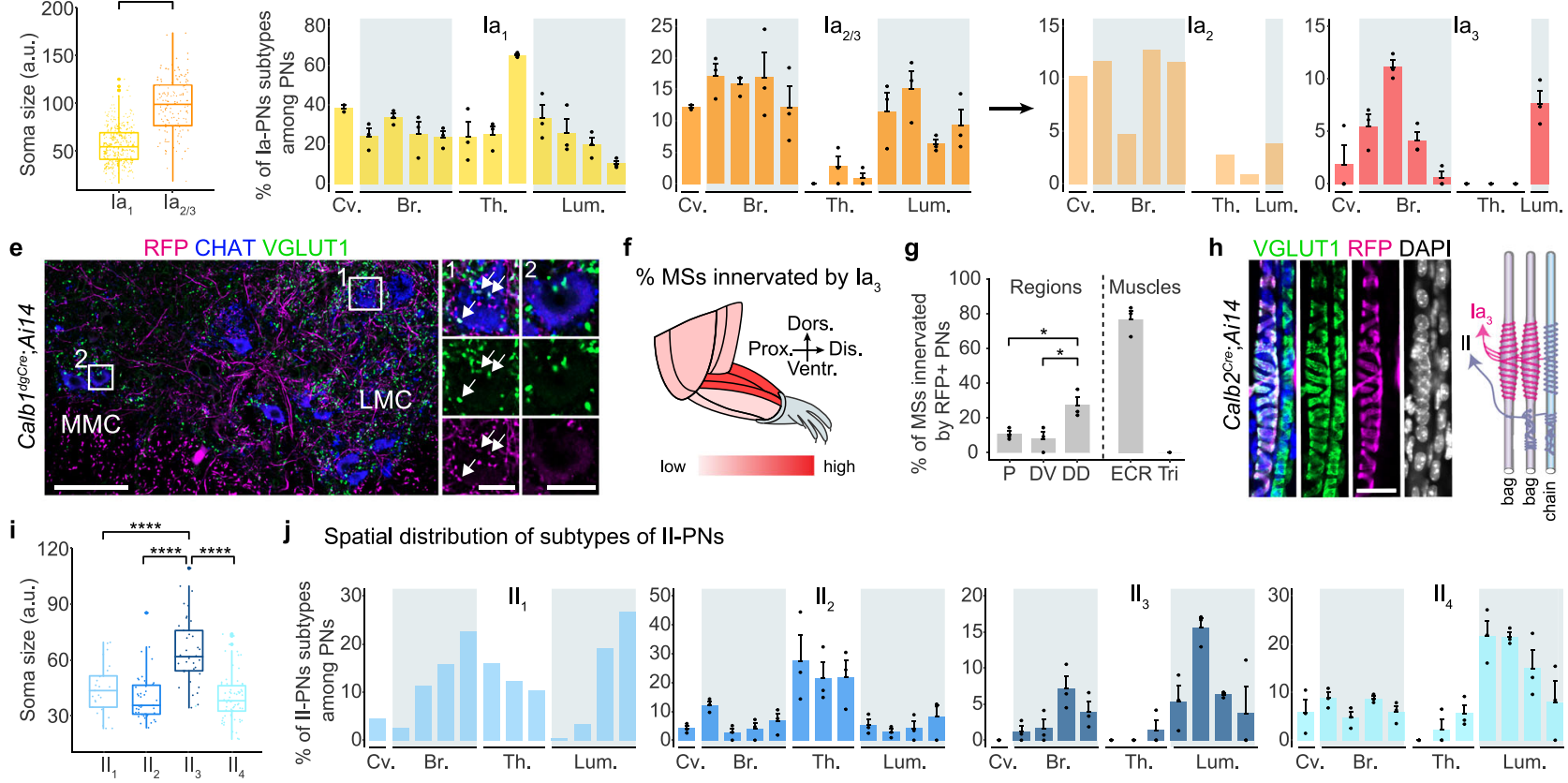

j Spatial distribution of subtypes of II-PNs

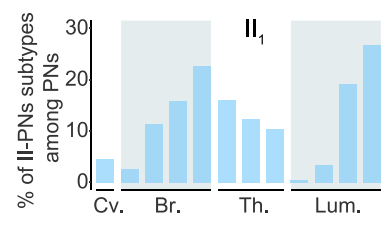

k Spatial distribution of PNs subtypes

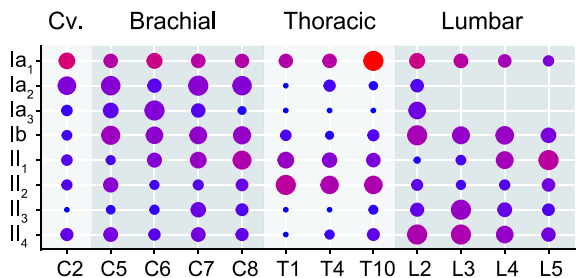

proportion to the highest represented DRG - $\bigcirc-1$ $\%$ among $\mathrm{PNs}$ in particular DRG
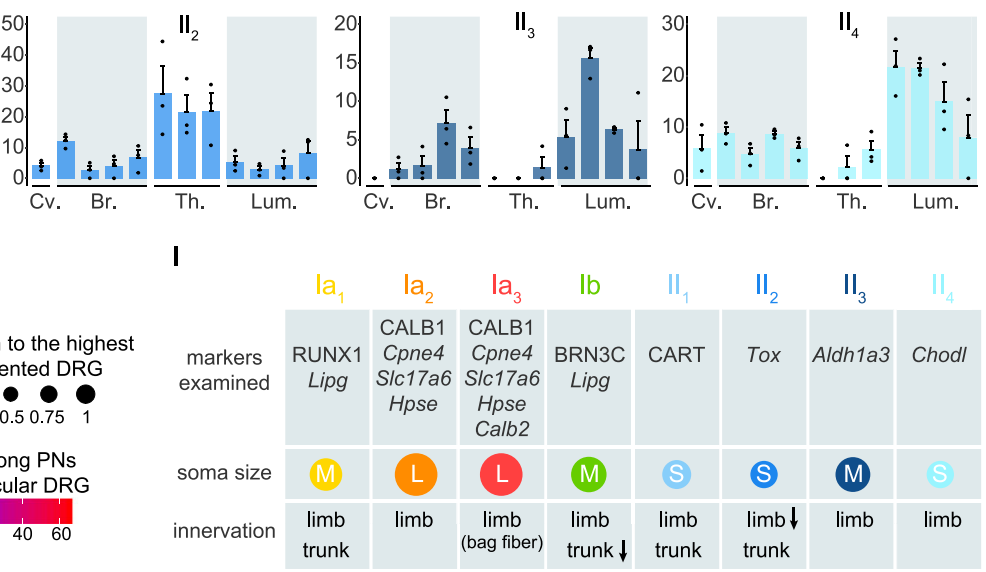

Fig. 3 Functional subdivisions of PNs. a Schematic depiction of the selected DRG (highlighted in red) to study the spatial distribution of PNs along the rostral-caudal axis of the trunk. $\mathbf{b}$ Spatial distribution of the three major types of PNs in representative DRG (see A) ( $n=3$ animals). Data are presented as mean \pm SEM; dots represent values from individual animals. See "Methods" for details. c Comparison of cross-nucleus soma sizes of la-PNs subtypes depicted in box and whisker plot. Lower and upper hinges: first and third quartiles; the horizontal line: median; the whiskers extend to the value no further than $1.5{ }^{*}$ IQR from the hinge; large dots: outliers. Each small dot represents a cell: $\mathrm{la}_{1}(n=342 \mathrm{cells}), \mathrm{la}_{2}$ and $\mathrm{la}_{3}$ together $(n=148$ cells). Two-tailed $t$-test, ${ }^{* \star \star *} p<0.0001$. d Spatial distribution of la-PNs subtypes in representative DRG (see A). Data are presented as mean \pm SEM ( $n=3$ animals); dots represent values from individual animals. See Methods for details. e Ventral spinal cord section of P54 Calb7dgCre;Ai74 mice labeled for CHAT (motor neurons) and VGLUT1 (sensory terminals), showing RFP+ sensory boutons onto motor neurons in LMC but not MMC. Scale bar: $100 \mu \mathrm{m}$. Scale bars of the micrographs: $20 \mu \mathrm{m}$. $\mathbf{f}$ Schematic depiction of the over-representation of $\mathrm{la}_{3}-\mathrm{PNs}$ innervation in the distal-dorsal region of the mouse forelimb. $\mathbf{g}$ Proportion of MSs innervated by RFP ${ }^{+}$afferents in P30 Calb2 Cre;Ai14 mice ( $n=3$ animals). P: proximal; DD: distal-dorsal; DV: distal-ventral; ECR: extensor carpi radialis; Tri: triceps. Data are presented as mean \pm SEM; dots represent values from individual animals. Two-tailed $t$-test, ${ }^{*} p<0.05$. $\mathbf{h}$ MS of P30 Calb2 ${ }^{C r e} ; A i 14$ mice stained for RFP, VGLUT1 and DAPI, showing that RFP+ afferents innervate only the nuclear bag but not chain fibers. Bag and chain fibers are distinguishable by their sizes and organization of nuclei: chain fibers have nuclei aligned in a chain, while bag fibers have many nuclei stacked in bags. Right panel: schematic representing the finding. Scale bar: $20 \mu \mathrm{m}$. i Quantification of cross-nucleus soma sizes of II-PNs subtypes depicted in box and whisker plot. Lower and upper hinges: first and third quartiles; the horizontal line: median; the whiskers extend to the value no further than 1.5 * $I Q R$ from the hinge; large dots: outliers. Each small dot represents a cell: $\|_{1}(n=25$ cells $), \|_{2}(n=43$ cells $), \|_{3}(n=37$ cells $), \|_{4}(n=65$ cells $)$. Two-tailed $t$-test, ${ }^{\star \star \star \star} p<0.0001$. j Spatial distribution of II-PNs subtypes in representative DRG (see a). Data are presented as mean \pm SEM ( $n=3$ animals); dots represent values from individual animals. See "Methods" for details. k Dot plot summarizing the spatial distribution of all PN subtypes. The size of the circle reflects how a subtype is distributed along the rostro-caudal axis where the presence of the subtype in each DRG is proportional to the highest represented DRG. The color intensity reflects the percentage of different subtypes among all PNs in the same DRG. I Table summarizing main characteristics of PN subtypes. The illustrated soma sizes of the subtypes are proportional to the average soma sizes of the respective PN subtypes observed in situ. Source data are provided as a Source Data file. 
a Mechanotransduction AP generation

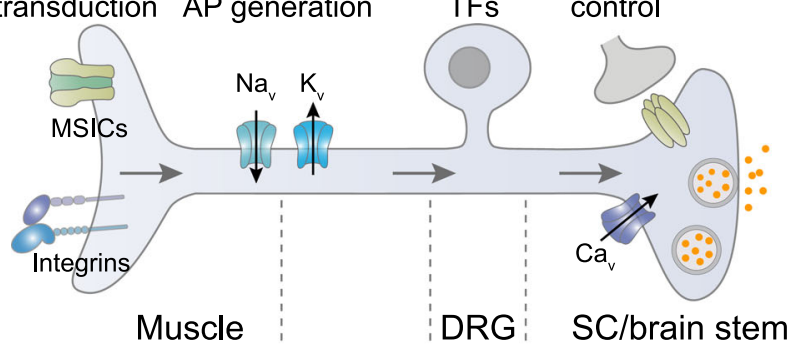

b

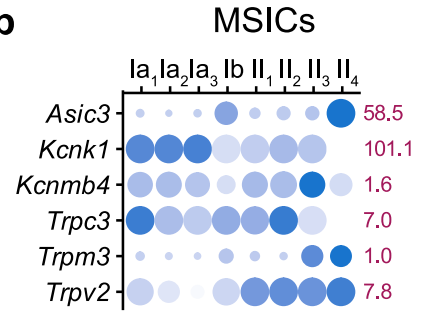

Mechanosensitive ion channels expressed by all subtypes: Asic1 Asic2 Kcnma1 Piezo2 Trpc1 Trpm2 Trpm4 Trpm7

e

Neurotr. transport

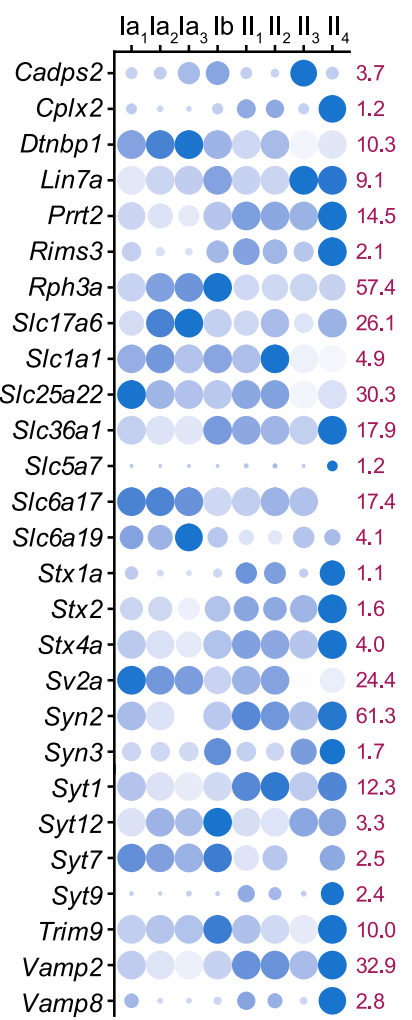

C

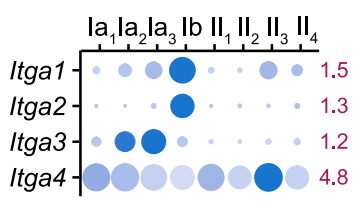

Neurotr. receptors

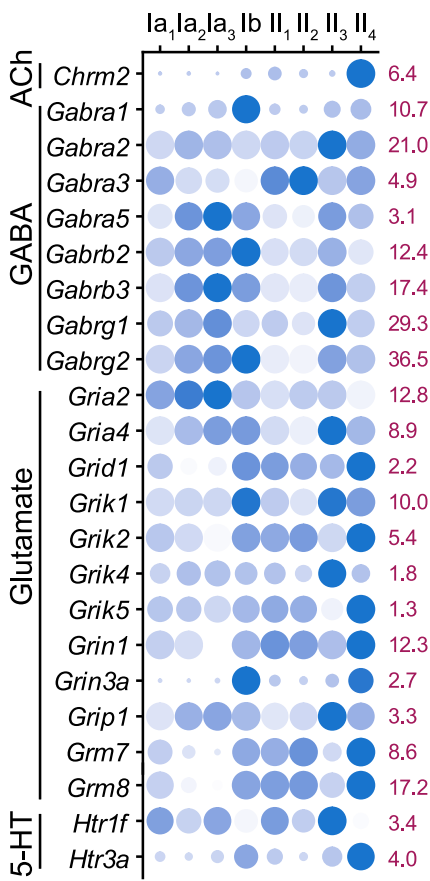

d

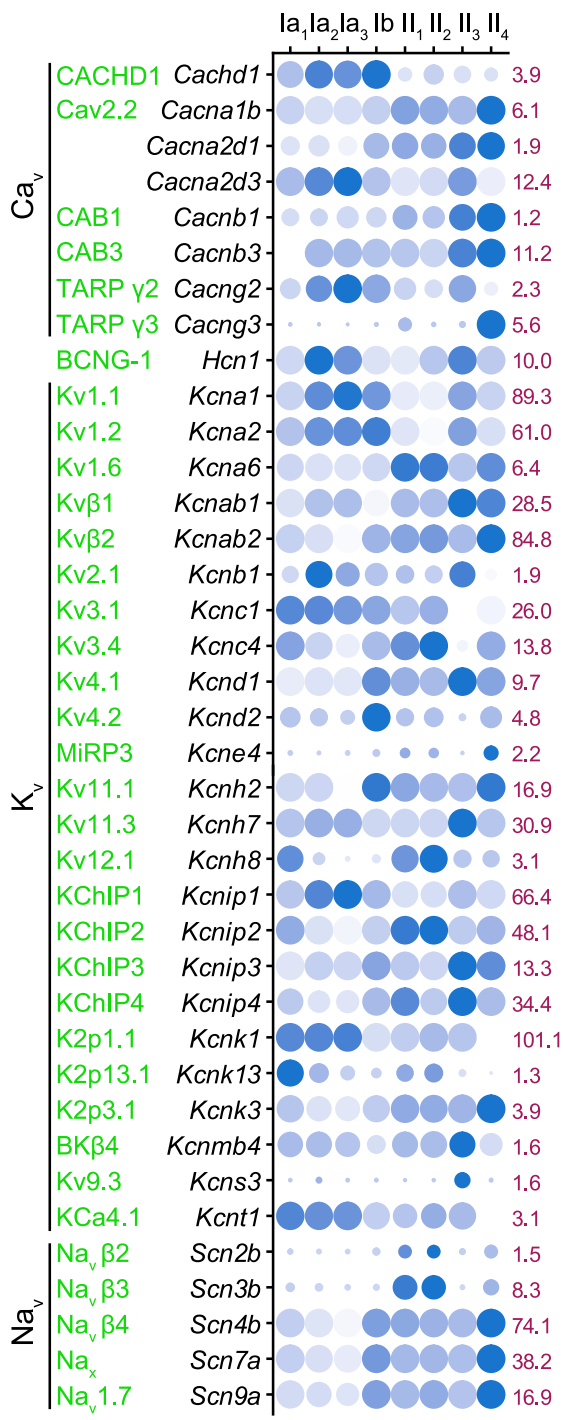

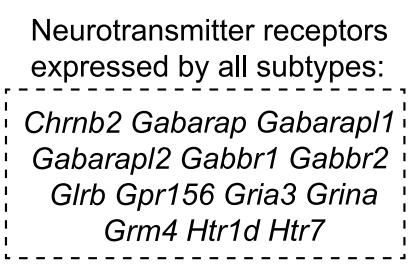

pct.exp

avg.exp

$1007550250 \quad-1.5 \quad 0.0 \quad 1.5$

Fig. 4 Unique molecular signatures of neuronal communication pattern in the PN subtypes. a Schematic depicting gene categories important for intrinsic electrical properties and input-output communication patterns of PNs. b-f Dot plots of differentially expressed genes for functional neuronal properties, including "mechanosensitive ion channels (MSICs)", "integrins", "voltage-gated ion channels (VGICs)", "neurotransmitter transport" and "neurotransmitter receptors". The highest mean of expression among the subtypes is specified in red. Protein names for VGICs are shown in green.

might require additional MSICs to acquire their unique mechanosensory property. Interestingly, the Ib-PNs unique expression of Itga1 and Itga2 (integrins $\alpha 1$ and $\alpha 2$ ) - the only known integrin subunits to heterodimerize with $\beta 1$ subunit to mediate cell surface binding to collagens ${ }^{24}$ - suggests that the mechano-activation of Ib-PNs might specifically involve $\alpha 1 \beta 1$ and $\alpha 2 \beta 1$ integrins (Fig. 4c). In this context, CHAD (chondroadherin), the main marker of Ib-PNs, is a secreted protein that interacts with both $\alpha 2 \beta 1$ integrins and collagens ${ }^{25}$ and is thus expected to mediate Ib nerve endings-matrix interactions.

Many voltage-gated sodium $\left(\mathrm{Na}_{\mathrm{v}}\right)$, calcium $\left(\mathrm{Ca}_{\mathrm{v}}\right)$, and potassium $\left(\mathrm{K}_{\mathrm{v}}\right)$ channels were differentially expressed in $\mathrm{PN}$ 
subtypes (Fig. 4d). Among them, $\mathrm{K}_{\mathrm{v}}$ channels and their accessory subunits exhibited the most diverse expression patterns (Fig. 4d), which would likely contribute to the regulation of various firing properties and signal integration of the different PN subtypes, as for the major types of somatosensory neurons ${ }^{26}$. Slc17a6 (VGLUT2), the glutamate transporter that mediates high fidelity of neurotransmitter release ${ }^{27}$, was highly enriched in $\mathrm{Ia}_{2}$ - and $\mathrm{Ia}_{3}-$ PNs (limb-innervating PNs) (Fig. 4e), suggesting increased synaptic strength with their postsynaptic partners, which might underlie a more effective and sensitive signal transmission required for the limb movements. Moreover, the gain and acuity of sensory inputs are modulated presynaptically by local spinal interneurons, in this context, many neurotransmitter receptors were also differentially expressed among PN subtypes with a few showing subtype-restricted expression (Fig. 4f). The observed discrete expression of neurotransmitter receptors in PNs likely contribute to controlling the sensitivity of the different proprioceptive sensory channels centrally but also to a possible discriminatory capacity of the proprioceptive system to focus on relevant afferent inputs, as shown between distinct sensory modalities ${ }^{28,29}$.

Postnatal emergence of PN subtypes. The discovery of the large diversity of PN subtypes leads us to ask when and how they emerge during development. Using scRNAseq of PNs at E16.5, a stage marked by already established connections with their peripheral targets and an ongoing central axon growth process to their spinal cord output neurons, three molecularly distinct clusters were identified (Fig. 5a). However, the transcriptomic profiles of E16.5 PNs exhibited large discrepancy from P54 PNs, making it challenging to connect those two stages with computational tools. Thus, to understand the classification of E16.5 PNs, we have identified marker genes for each cluster (cl.1: Vstm2b; cl.2: Tnfaip8l3; cl.3: Doc2b) and used them to label the clusters in vivo (Fig. 5b, c). In order to identify Ia-PNs among the three major clusters, we injected rhodamine dextran (Rh-Dex) in the MN region of the brachial ventral spinal cord of E16.5 embryos to retrogradely target Ia-PNs (Fig. 5d-f) (see "Methods" for details). After ex vivo incubation, both pre-motor interneurons in the spinal cord and Ia-PNs in the DRG were selectively labeled by Rh-Dex (Fig. 5e, f) ( $90 \%$ of Rh-Dex ${ }^{+}$DRG cells were PNs). Interestingly, while Tnfaip $813^{+}, V_{s t m} 2 b^{+}$and $D o c 2 b^{+} \mathrm{PNs}$ represented 20.3, 37.0 and $30.0 \%$ of PNs in brachial DRG, respectively, the Tnfaip $83^{+}$population was significantly overrepresented (68.0\%) in Rh-Dex ${ }^{+}$PNs (Fig. 5g), suggesting the cl.2 to be Ia-PNs. A small number of Vstm $2 b^{+}$and Doc $2 b^{+}$cells were also traced in our experiments (Fig. 5g). This could be explained by the diffusion properties of the dextran that might be taken up by few nerve endings of other PNs terminating in the vicinity of the ventral horn of the spinal cord at this early stage (see "Methods" for details), but also by the incomplete cell-type specificity of the markers used, which at this stage showed enrichment but not unique expression in a given cluster (Fig. 5b). To genetically trace the Doc $2 b^{+}$PNs lineage (cl.3), we generated a transgenic mouse driver line Doc $2 b^{d d C r e}$, expressing trimethoprim (TMP)-inducible ddCre under the control of the endogenous Doc $2 b$ locus and where Cre recombinase is fused to the destabilizing DHFR domain, which can be transiently stabilized by the administration of TMP (Fig. 5h) ${ }^{30}$. Using Doc2 $b^{d d C r e} ;$ Ail 4 mouse, we genetically traced the Doc $2 b^{+}$cells from E16.5 and examined them postnatally with adult PN markers (Fig. 5i). Notably, we observed that almost $90 \%$ of Ib-PNs $\left(\mathrm{BRN}^{+} \mathrm{C}^{+}\right)$were labeled by RFP at brachial level (Fig. 5i), while less than $30 \%$ of Ia- and II-PNs were positive, strongly suggesting that Doc2b marked mostly the Ib-PNs at E16.5. The small fraction of traced
Ia- or II- PNs in Doc $2 b^{d d C r e}$; $A i 14$ mouse might be the result of a baseline recombination often seen in $d d C r$ mouse lines (see Methods for details). Additionally, at E16.5, Doc2 $b^{+}$PNs were found specifically enriched in DRG innervating the limbs (Fig. 5j), another characteristic of Ib-PNs (Fig. 3b). Together, these data suggest that cl.2 and cl.3 of E16.5 PNs identify Ia- and Ib-PNs, respectively, and the remaining cl.1 thus most likely represented II-PNs (Fig. 5k). We have also provided a list of the transcription factors expressed in each PN type at this critical developmental stage (Supplementary Fig. 5a), which will help future investigations of the molecular programs that control the differentiation and connectivity patterns of PNs during early development.

While the three majors clusters could be split further into subclusters, those were only found at this early developmental stage and a gene ontology analysis of these subdivisions' markers revealed a high enrichment in terms involved in synapse assembly, cell adhesion and axon guidance. This suggests that the upregulation and differential expression of genes necessary for the assembly of sensorimotor circuit around E16.5 might generate transcriptional diversity resulting in transient subdivisions amongst the major cell types, as recently suggested for the developing visual system in drosophila ${ }^{31}$. Further developmental studies will be necessary to explain the biological basis of the subcluster diversity of PNs at this stage (Supplementary Fig. 5b, c).

We then asked whether PNs diversification begin during the early postnatal period, when activity-dependent mechanisms remodel neural circuits $^{32,33}$. For this, we generated scRNAseq data of P5 PNs, when the pups still exhibit immature locomotor behavior, and identified 6 molecularly distinct clusters at this stage (Fig. 5l). The major PN types Ia (cl.1), Ib (cl.2) and II (cl.3,4,5,6) were already identified by their overall transcriptomic similarity to adult PN types and expression of adult markers (Fig. 51 and Supplementary Fig. 6a, b). Interestingly, II-PN subtypes were already present at this stage and expressed corresponding adult markers (Fig. $5 \mathrm{~m}$ ), while Ia-PNs were not yet clearly subdivided into the subtypes found in adult (Fig. $5 \mathrm{~m}$ ). In addition, P5 Ia-PNs expressed many Ib- or II-PN markers, while those were reduced or absent in adult Ia-PNs (Supplementary Fig. 6c). Specifically, many genes related to neuronal functions (e.g., ion channels and neurotransmitter receptors), which were expressed in P5 Ia-PNs were later downregulated at P54 (Supplementary Fig. 6d), likely reflecting that the subtype characters of Ia-PNs might be sharpened through repressing genes that are nonspecific or no longer required for their mature neuronal activities.

Taken together, these observations suggest that the three main types of PNs (Ia, Ib, and II) differentiate simultaneously with the formation of the sensorimotor circuit during embryogenesis (Fig. 5k), while PN subtypes emerge postnatally (Fig. 5n). The diversification of the II-PNs into four subtypes around birth could result from changes in the environment, possibly including motor or spontaneous activity. Ia-PNs diversification into three subtypes might require further sensory experience through patterned motor activity and involve trimming away nonspecific neurotransmission-related genes of the alternative adult PN subtypes.

Activity-driven plasticity of adult Ia-PNs. The observation of the relatively late differentiation of Ia-PNs led us to investigate whether the identity of Ia-PN subtypes remains versatile in adult life following changes in sensorimotor activity. To test this, we used motor skill learning that has been intensely studied in the realm of neuroplasticity ${ }^{34-36}$. More specifically, we assessed the composition of Ia-PN subtypes in DRG of wild-type mice with 


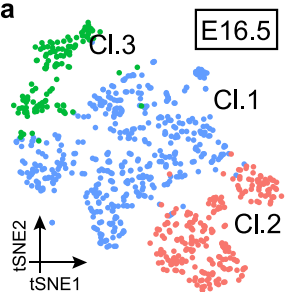

b

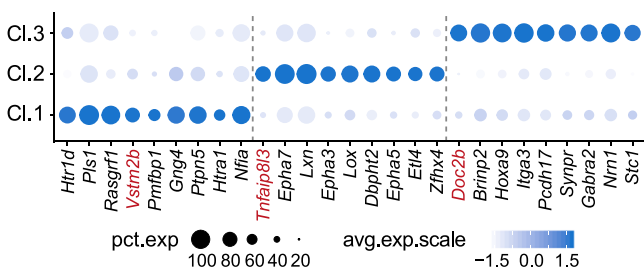

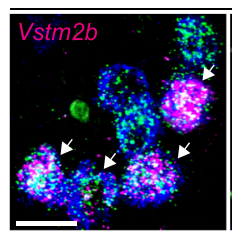

Pvalb Runx3

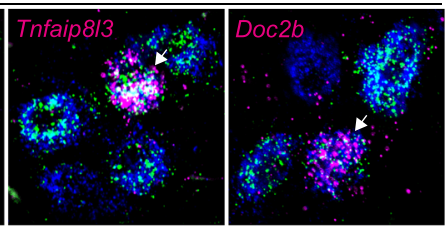

d
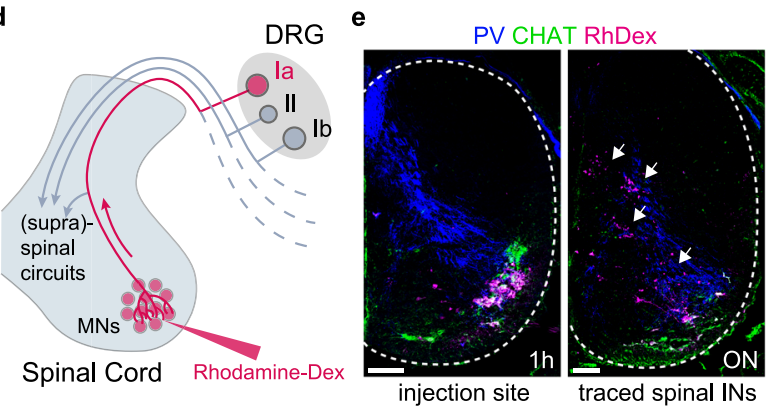

PV RUNX3 RhDex
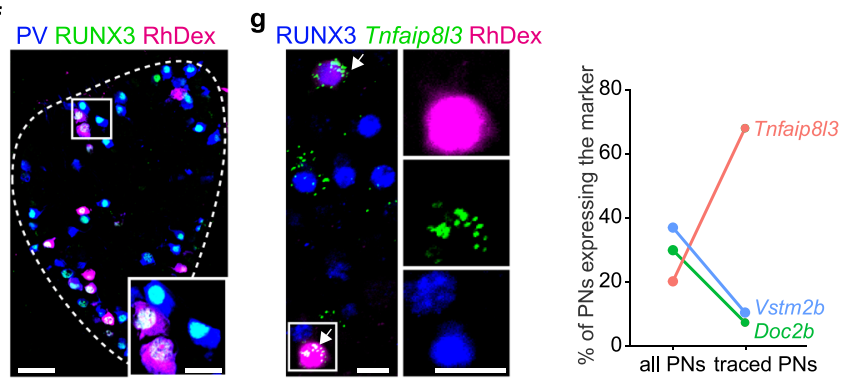

$\mathbf{h}$

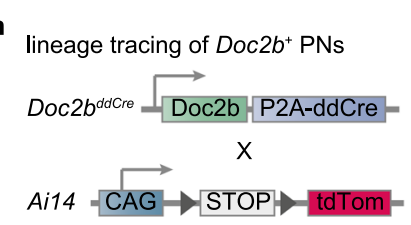

TMP injection at E16.5
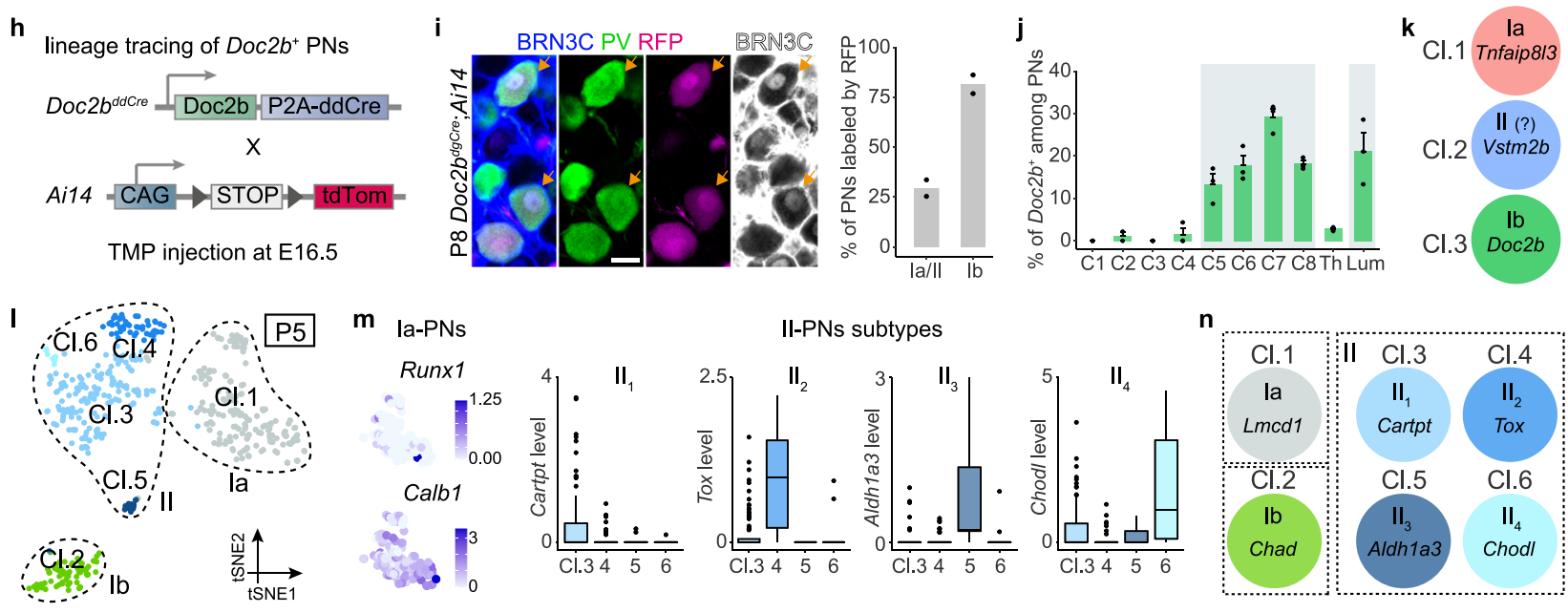

Fig. 5 Emergence of PN subtypes during development. a Three molecularly distinct clusters of E16.5 PNs shown by tSNE. b Dot plot illustrating examples of marker genes in each cluster. $\mathbf{c}$ In vivo validation of the three E16.5 PN populations by RNAscope using the identified markers (magenta) and PN markers Pvalb and Runx3. Scale bar: $20 \mu \mathrm{m}$. d Strategy to retrogradely label la-PNs in E16.5 embryos. e Left: transverse section of spinal cord $1 \mathrm{~h}$ post-injection stained for PV and CHAT, showing the injection site in the motor neurons area. Right: transverse section of spinal cord over night (ON) post-injection stained for PV and CHAT, showing the retrogradely labeled pre-motor spinal interneurons. Scale bars: $100 \mu \mathrm{m}$. f DRG section from embryo $6 \mathrm{~h}$ postinjection stained for PN markers PV and RUNX3, showing the retrogradely labeled la-PNs. Scale bar: $50 \mu m$. Scale bar of the micrograph: $20 \mu m$. g Retrogradely labeled DRG section stained for PN marker RUNX3 (immunostaining) and subtype marker Tnfaip8/3 (RNAscope). Right: Proportion of the three PN populations among all PNs $\left(P v a l b^{+} / R_{u n} \times 3^{+}\right)$and retrogradely traced PNs from experiment in $\mathbf{d}(n=4$ embryos for Tnfaipl3 and Doc $2 b, n=3$ embryos for Vstm2b). Scale bar: $20 \mu \mathrm{m}$. Scale bar of the micrograph: $20 \mu \mathrm{m}$. h Strategy to genetically trace the Doc $2 b^{+}$PNs lineage (TMP: trimethoprim). i P8 DRG section from Doc2 $b^{d d C r e}$;Ai14 mice injected with TMP at E16.5. The sections were stained for PN marker PV and postnatal Ib-PN marker BRN3C. The staining of BRN3C in gray scale is pseudo-coloring from blue. Scale bar: $20 \mu \mathrm{m}$. Right: proportion of la/II-PNs and Ib-PNs labeled by RFP, respectively, presenting enriched tracing of $\mathrm{Ib}-\mathrm{PNs}\left(n=2\right.$ animals); dots represent values from individual animals. $\mathbf{j}$ Spatial distribution of Doc2 $b^{+} \mathrm{PNs}$ in representative DRG of E16.5 embryos. Data are presented as mean \pm SEM ( $n=3$ animals). Data for Th. and Lum. regions represent an average of the proportion observed in segments T2-T12 and L1-L5, respectively. $\mathbf{k}$ Correspondence between PN types and the clusters identified by scRNAseq at E16.5. I tSNE of PNs depicting molecularly distinct clusters at P5. $\mathbf{m}$ Box and whisker plots showing the marker expression of 4 II-PN subtypes at P5, while la-PN subtypes were indistinguishable by their subtype markers at this stage (e.g., Runx1, Calb1). Lower and upper hinges: first and third quartiles; the horizontal line: median; the whiskers extend to the value no further than $1.5{ }^{*}$ IQR from the hinge; large dots: outliers. For the box plots, $\mathrm{Cl} .3(\mathrm{n}=108), \mathrm{Cl} .4(n=96), \mathrm{Cl} .5(n=14), \mathrm{Cl} .6$ $(n=11)$. $\mathbf{n}$ Correspondence between PN types and the clusters identified by scRNAseq at P5. Source data are provided as a Source Data file.

access to free-wheel running for 4 weeks, which is known to induce experience-dependent plasticity in the central nervous system $^{36-38}$. During the entire training period, running (Run) and sedentary (Sed) mice were single housed in enhanced environment: large rat cage, wooden sticks, nest and free access to a running wheel (Fig. 6a). The wheel was in locked mode in the sedentary condition (Fig. 6a). Each exercised mouse spent substantial amount of time on the wheel and ran on average $8 \mathrm{~km} /$ day. No gain of weight in the running group compared with the sedentary groups showed the efficiency of running (Fig. 6b). At the end of the training period, mice were sacrificed and analyzed for PNs phenotype. The overall proportion of Ia-PNs (among all PNs) (Fig. 6c) and their total number (raw counts, normalized to sedentary condition: Sed $1 \pm 0.13$ and Run $0.98 \pm 0.005)$ in brachial DRG of sedentary and running animals were identical. Importantly, in the running condition we observed a decrease in 
a

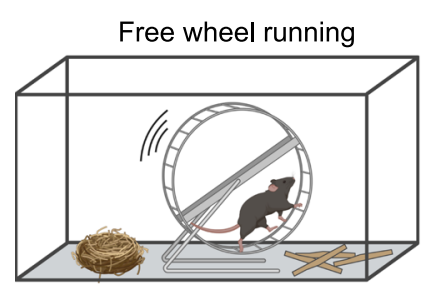

adult mice in voluntary running experiment

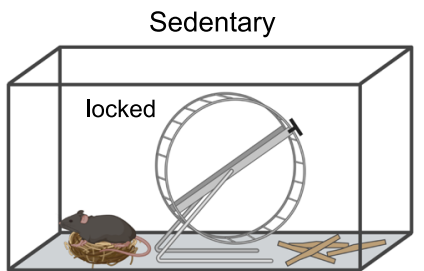

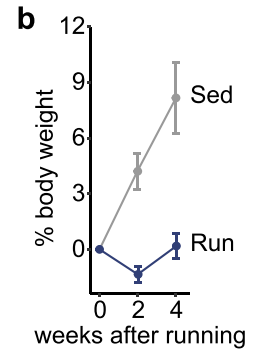

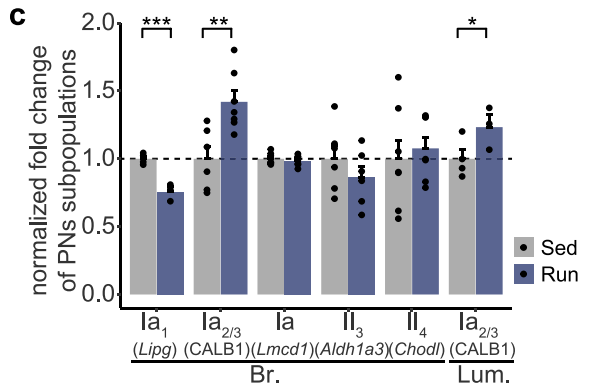

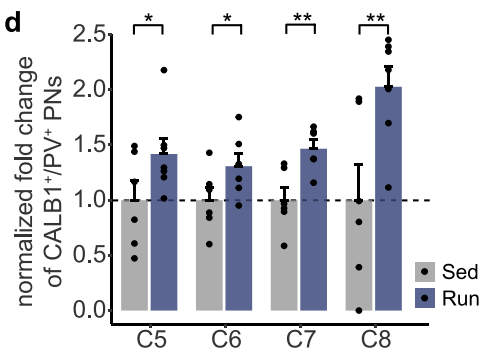

f

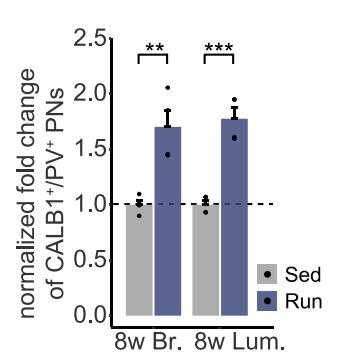

e

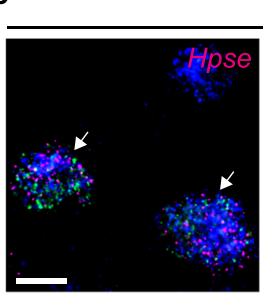

Whrn Calb1 g

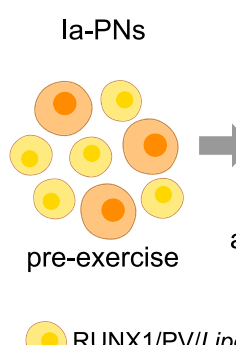

RUNX1/PVILipg

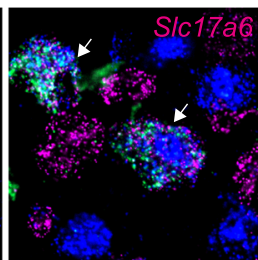

h
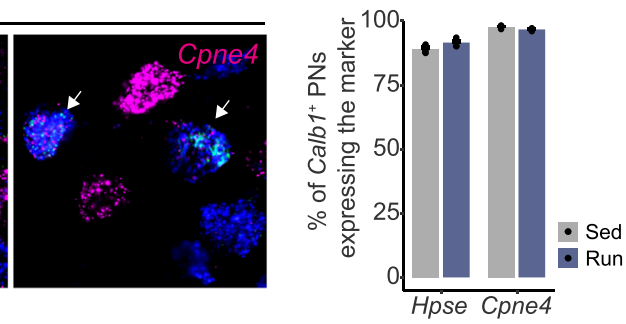

Diversification of PNs subtypes

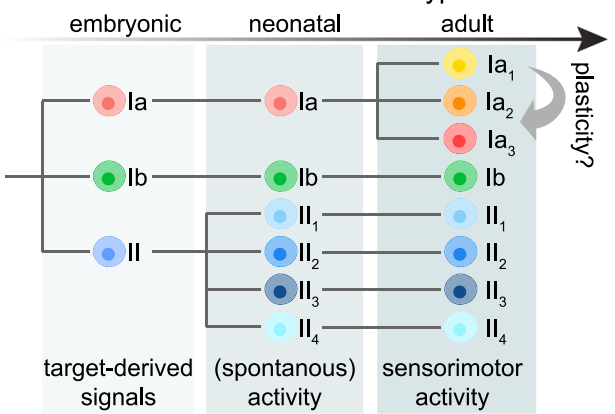

Fig. 6 Activity-driven plasticity of adult la-PN subtypes. a Schematic depiction of the housing environment during the voluntary running experiment. b Percentage of weight change in sedentary (Sed) and free wheel running (Run) groups. Data are presented as mean \pm SEM ( $n=7$ animals for both groups). c Fold change of proportion of PN subtypes in 4-week running group compared with sedentary group: $\mathrm{Lipg}^{+} / \mathrm{Whrn}^{+} \mathrm{cells}\left(\mathrm{la}_{1}\right.$ ) among Whrn $\mathrm{am}^{+}$cells,

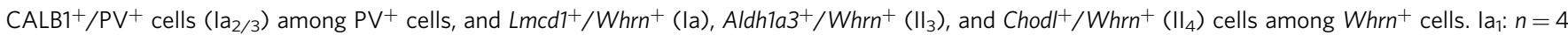
animals for sedentary group and $n=3$ animals for running group; $\mathrm{la}_{2 / 3}(\mathrm{Br}$.): $n=6$ animals for sedentary group, $n=7$ animals for running group; la: $n=7$ animals for both groups; $\|_{3}: n=6$ animals for both groups; $\|_{4}: n=7$ animals for both groups; $I_{2 / 3}$ (Lum.): $n=4$ animals for sedentary group, $n=3$ animals for running group. Data are presented as mean \pm SEM; dots represent values from individual animals. One-tailed $t$-test, ${ }^{\star} p<0.05$, ${ }^{\star \star} p<0.01,{ }^{\star \star \star} p<0.001$. d Fold change of proportion of $\mathrm{CALB} 1^{+} / \mathrm{PV}+\mathrm{PNs}$ in individual brachial segments in 4-week running group compared with sedentary group $(n=7$ animals). Data are presented as mean \pm SEM; dots represent values from individual animals. One-tailed $t$-test, ${ }^{\star} p<0.05$, ${ }^{\star \star} p<0.01$. e RNAscope images showing the expression of other $\mathrm{la}_{2 / 3}-\mathrm{PN}$ markers (magenta) in Calb1+ $\mathrm{PNs}\left(\mathrm{Whrn}^{+}\right)$on the brachial DRG sections of the running mice. Scale bar: $20 \mu \mathrm{m}$. Right: percentage of $\mathrm{Calb}^{+}{ }^{+}$PNs expressing other $\mathrm{la}_{2 / 3}$ markers in sedentary and running groups, respectively $(n=4$ animals). Data are presented as mean \pm SEM; dots represent values from individual animals. $\mathbf{f}$ Fold change of proportion of CALB1 ${ }^{+} / \mathrm{PV}^{+} \mathrm{PNs}$ in 8 -week running group compared with sedentary group ( $n=4$ animals). Data are presented as mean \pm SEM; dots represent values from individual animals. One-tailed $t$-test, ${ }^{\star \star} p<0.01,{ }^{\star \star \star} p<0.001$. g Schematic summarizing the results from the voluntary running experiment. h Model of the diversification of PN subtypes. Br: brachial DRG (C5-8); Lum: lumbar DRG (L2-5). Source data are provided as a Source Data file.

the proportion of $\mathrm{Lipg}^{+} / \mathrm{Whrn}^{+}$PNs (labeling $\mathrm{Ia}_{1}$-PNs) and an increase in $\mathrm{CALB}^{+} / \mathrm{PV}^{+}$PNs (labeling $\mathrm{Ia}_{2 / 3}-\mathrm{PNs}$ ) at limb level (Fig. 6c, d). In contrast, analysis of II-PNs in DRG from the same animals did not reveal any change in subtype composition (Fig. 6c), suggesting that this type of neuronal plasticity mediated by increased locomotor activity is specific to Ia-PNs. To confirm the observed changes in Ia-PNs subtype composition, we tested other $\mathrm{Ia}_{2 / 3}-\mathrm{PN}$ markers such as Hpse, Slc17a6 and Cpne4. In the running mice, virtually all $\mathrm{Calb} 1^{+} \mathrm{PNs}$ were found to also express these $\mathrm{Ia}_{2 / 3}-\mathrm{PN}$ markers (Fig. 6e), indicating that the transcriptional dynamic in Ia-PNs following sustained exercise training is not limited to a single-marker gene. After 8-week training, the proportional increase of $\mathrm{Ia}_{2 / 3}$-PN markers was further enlarged to 70 and $77 \%$ at brachial and lumbar level, respectively (Fig. 6f). Altogether, these data suggest an increase in a $\mathrm{Ia}_{2 / 3}-\mathrm{PN}$ identity after running exercise at the expense of a $\mathrm{Ia}_{1}-\mathrm{PN}$ identity (Fig. 6g). As described earlier, among PNs, the $\mathrm{Ia}_{2 / 3}$ subtypes would represent highly dynamic populations. Thus, the observed plasticity of Ia-PNs with versatile subtype composition might serve as one mechanism of the adult proprioceptive system to adapt to changing motor behavior. Whether this plasticity is controlled by activity-dependent programs or target-derived or systemic factors, e.g., cytokines or growth factors (from muscles or blood), is awaiting to be addressed.

\section{Discussion}

The first main insight from our results is the demonstration of an extensive diversity of PNs with discrete anatomical organization and unique genetic identities, a molecular and cellular diversity long searched for but never observed. This classification of PNs suggests that the proprioceptive system is endowed with a vast array of PN subtypes that are necessary to extract and transmit the various features of muscle activities across the body. The 
second main result is the late emergence of the dynamic subtypes of the Ia-PNs and their plasticity in the adult in the form of changing subtype identity after sustained exercise training. This plasticity suggests neuronal individuality in the nervous system to adapt its performance to changing environment.

Adult PNs have long been recognized to comprise three functional types (Ia-, Ib-, and II-PNs) based on their anatomy and electrophysiological properties ex vivo, however, molecular tools to distinguish them have been lacking, preventing research into their individual functions in vivo, especially their acute modulation in adult. In this study, we identified eight discrete subtypes among the three major types, with their respective molecular markers, hence providing tools for direct (intersectional) genetic strategies to address their functional connectivity and roles in vivo. Importantly, our comprehensive molecular delineation of PN subtypes also provides a large set of functional genes that are predictive of the biophysical and physiological features of PNs and will stimulate future investigations in the field.

The current view on the organization of the proprioceptive feedback describes an elementary modular organization, where three types of PNs each relay the muscle length, speed and force, respectively, omitting the fact that muscles are distinct in their functionality and that their activities cover a diverse range of sensitivity. Thus, our discovery of the eight functional subtypes of PNs extend this view and demonstrate a more sophisticated organization where distinct $\mathrm{PN}$ subtypes serve only selective muscle targets (Fig. 31), and would differ in their dynamic sensitivity, which would increase for instance from $\mathrm{Ia}_{1}-$ to $\mathrm{Ia}_{3}-\mathrm{PNs}$. These differences could participate in the detection and transmission of a large array of sensory stimuli. How these various sensory inputs are integrated and processed centrally for controlling motor outputs are intriguing topics for future studies, which can now be envisaged using our molecular tools. Moreover, considering the executive role of the proprioceptive feedback in directing restoration of the motor function after spinal cord injury $^{2}$, our findings will also help developing experimental strategies to study and modulate specific MS feedback channels for therapeutic intervention.

Our data indicate that diversification into these PN subtypes follows a three-step model, where an early genetic programming establishes PN cardinal identity (after neurogenesis) 39,40 that is refined into PNs types following innervation ${ }^{41}$, and which later diversify into several subtypes postnatally (Fig. 6h). The observation that the diverse PN subtypes found in adult could be identified only postnatally favors the influence of neuronal activity in driving their later diversification program ${ }^{42,43}$. Moreover, the observed delayed diversification of Ia-PN subtypes suggests experience-dependent plasticity involving a tight interplay between sensorimotor experience and neuronal identity. Remarkably, we further demonstrate that the substantial plasticity of Ia-PN subtypes persist in the adult by changing subtype identity following sustained running paradigm that has been associated with motor skill learning ${ }^{36}$. This reveals another level of neuroplasticity given that most studies on (non-pathological) neuroplasticity in adult has been focusing on cortical and subcortical areas and demonstrated structural adaptations and/or local changes in synaptic strength. Future studies will be necessary to understand the physiological relevance of such plasticity and whether the observed changes reflect a real switch in cell type and if it is reversible or remains stable over time. It will also be interesting to address the influence of the environment in generating neuronal individuality in other regions of the peripheral nervous system, and how this heterogeneity might correlate with performance in sensory processing functions and eventually individual behavioral differences.

\section{Methods}

Mouse lines. All animal experiments were approved by the local ethical committee (Stockholms Norra djurförsöksetiska nämnd, Sweden) and conducted following the ethical guidelines described in the Swedish Animal Agency's Provisions and Guidelines for Animals Experimentation Recommendations. Mice were housed in cages in groups, with food and water ad libitum, under $12 \mathrm{~h}$ light-dark cycle conditions. PV Cre; Ail4 mice were crossed from PVCre (from The Jackson Laboratory $^{44}$ ) and Ai14 (from The Jackson Laboratory ${ }^{45}$ ), and used to genetically label PNs for scRNAseq experiments. Egr $3^{W G A}$ mice ${ }^{19}$ were used to specifically label MSs-innervating PNs. ChAT ${ }^{\text {Cre }} ; R G T$ mice were crossed from $C h A T^{\text {Cre }}$ (from Ole Kiehn $\mathrm{lab}^{46}$ ) and $R G T$ (from The Jackson Laboratory ${ }^{20}$ ), and used to specifically express avian receptor protein TVA and rabies glycoprotein in MNs for rabies virus infection. Calb2 ${ }^{\mathrm{Cre}} ; \mathrm{Ai14}$ mice $^{47}$ were used to genetically label $\mathrm{Calb2}^{+}$cells in DRG for neuroanatomical tracing. Calb1 ${ }^{\text {dgCre; }}$ Ail4 mice were crossed from Calb1 ${ }^{d g C r e}$ (from The Jackson Laboratory ${ }^{48}$ ) and Ai14, and used to genetically label and trace the nerve endings of adult Calb1 ${ }^{+}$PNs. Doc $2 b^{d d C r e} ; A i 14$ mice were crossed from Doc $2 b^{d d C r e}$ (generated in collaboration with Gurumurthy lab) and $A i 14$, and used for lineage tracing of embryonic Doc $2 b^{+}$PNs. C57BL/6J mice were received from The Jackson Laboratory (stock \#000664) and used for most experiments unless otherwise specified. Primers used for genotyping of the mouse strains are listed in Supplementary Table 1.

Generation of Doc $\mathbf{2 b}^{\text {ddCre }}$ mouse model. The Doc $2 b^{d d C r e}$ mouse model was created using the Easi-CRISPR method ${ }^{49,50}$. Briefly, a long single-stranded DNA donor containing P2A-ddCre cassette was used as a repair template to insert immediately before the stop codon of Doc $2 b$ gene. The donor DNA was injected into pronuclei derived from $C 57 B L 6 / J$ mice along with CRISPR Ribonucleoproteins (a synthetic guide RNA and Cas9 protein mix). The final concentrations of components in ctRNP (crRNA + tracrRNA) preparations were $10 \mathrm{ng} / \mu \mathrm{l}$ of guide RNA and $10 \mathrm{ng} / \mu \mathrm{l}$ of Cas 9 protein. The ssDNA donor was mixed with ctRNP complexes at final concentration $10 \mathrm{ng} / \mu \mathrm{l}$ and the final injection mixes were passed through Millipore Centrifugal Filter units (EMD Millipore, cat\#UFC30VV25) and spun at $21,000 \times g$ for $5 \mathrm{~min}$ at room temperature. Superovulation, zygote isolation, pronuclear microinjection, surgical transfer of the zygotes into pseudo-pregnant mice were performed following the standard protocols described previously ${ }^{50}$. The live offspring were genotyped using 5' and 3' Junction PCRs, standard strategies used for identifying knock-in cassettes ${ }^{50}$. The entire region of the knock-in cassette was verified by Sanger sequencing. The model was generated at the mouse genome engineering core facility, university of Nebraska medical center. The line was shipped to Karolinska Institutet where it was rederived in C57BL6/J mice using in vitro fertilization.

Trimethoprim treatment. On the day of intraperitoneal (IP) injection, Trimethoprim (TMP) (Sigma-Aldrich, cat\#T7883) was dissolved in dimethyl sulfoxide (Fisher Scientific, cat\#10206581) at concentration of $50 \mathrm{mg} / \mathrm{ml}$ and diluted 1:3 into $0.9 \%$ saline solution to obtain working solution. For Calb1 $1^{d g C r e}$;Ai14 strain, P30 mice were treated with TMP $(100 \mu \mathrm{g} / \mathrm{g}$ b.w.) on 3 consecutive days. Two weeks after the injection, the mice were anesthetized by isoflurane and perfused for tissue collection. For the tracing of Doc $2 b^{+}$cells, pregnant females were treated with single dose $(125 \mu \mathrm{g} / \mathrm{g}$ b.w.) of TMP at E16.5. The P8 pups were anesthetized by isoflurane and perfused for tissue collection. Owing to the nature the TMPinducible $d d C r e$ mouse lines, it is possible that for some genes that are highly expressed, an excess amount of destabilized Cre is produced that exhausts the degeneration capacity, leading to certain level of recombination in the absence of TMP $^{26}$. On account of this, we have observed that in the absence of TMP Doc2$b^{d d C r e} ;$ Ai14 mice displayed recombination in $13.5 \%$ of PNs, among which the proportion of $\mathrm{Lmcdl}^{+} / \mathrm{Whrn}^{+}, \mathrm{Fxyd}^{+} / \mathrm{Whrn}^{+}$and $\mathrm{Chad}^{+} / \mathrm{Whrn}^{+}$was $43.5 \%$, $29.0 \%$ and $29.5 \%$, respectively.

Retrograde labeling of la-PNs. For the retrograde labeling of adult Ia-PNs, $C h A T^{\text {Cre }}$ mice were crossed with $R G T$ mice to obtain the ChAT ${ }^{C r e} ; R G T$ strain (heterozygous for both alleles). Viral injections with EnvA- $\Delta$ G-eGFP were performed in multiple areas of the lumbar cord once the animals became adult (60 days old). For the injections, the animal was anesthetized with isoflurane and shaved on the dorsal side. Sodium iodine was applied on the shaved skin. Eye ointment was applied on the eyes to prevent de-hydration. After skin incision, the spinal column with the lumbar segments were localized (from the 12th thoracic vertebra to the 1st lumbar vertebra) and fixed with two holders on the left and right side to prevent movement (mostly due to respiration). A vertical incision was made in front of the spinous process followed by the cut of the Ligamentum Flavum between the two vertebral bodies. At this point the spinal cord appeared. This procedure was repeated three times to expose and inject three different lumbar segments along the spinal cord. Then, the EnvA- $\Delta \mathrm{G}-\mathrm{eGFP}$ was bilaterally injected $(200 \mathrm{~nL}$ per segment, $0.1 \mu \mathrm{l} / 1 \mathrm{~min}$.), using a glass pipette (tip of about $80 \mu \mathrm{m}$ of diameter). The injection of the virus was complete in about $15 \mathrm{~min}$ for the three segments. Once the injections were done, the muscles and the skin were sutured. Animals were given Buprenorphine $(0.1 \mathrm{mg} / \mathrm{kg})$ and Carprofen $(5 \mathrm{mg} / \mathrm{kg}) \mathrm{sub}-$ cutaneously for 2 to 5 days. Infections or excessive loss of body weight in the postsurgery period was indication of experiment termination. 
For the retrograde labeling of E16.5 Ia-PNs, the anterior longitudinal ligaments of the brachial region were removed to expose the ventral spinal cord, $1 \%$ Rhodamine-Dextran (Life Technologies, cat\#D-3308) was injected into the ventral horn, which is innervated by the Ia-PNs. Preparations were incubated for $6 \mathrm{~h}$ in DMEM-F12 medium aerated with $5 \% \mathrm{CO}_{2}$ in $95 \% \mathrm{O}_{2}$ (Carbogen) before fixation. $20 \%$ of PNs $\left(\mathrm{Pvalb}^{+} / \mathrm{Run}^{+} 3^{+}\right)$were Rho-Dex ${ }^{+}$, while $90 \%$ of Rho-Dex ${ }^{+}$cells were PNs, showing the high efficiency and specificity of using this strategy to backfill Ia-PNs.

Immunohistochemistry and in situ hybridization (RNAscope). Postnatal mice were transcardially perfused with $20 \mathrm{ml}$ phosphate-buffered saline (PBS) and $20 \mathrm{ml}$ $4 \%$ PFA. For immunohistochemistry, tissues were dissected and post-fixed in $4 \%$ paraformaldehyde (PFA, AH diagnostics, cat $\#$ sc-281692) at $4{ }^{\circ} \mathrm{C}$ according to the sizes (P14 DRG: 1 h, P54 DRG: 1.5 h, P7 spinal column: 6 h, P54 forelimb: O/N). For RNAscope experiments, tissues were always post-fixed O/N in $4 \%$ PFA at $4{ }^{\circ} \mathrm{C}$. The tissues were then washed three times with PBS and cryoprotected in $20 \%(\mathrm{O} / \mathrm{N}$ at $\left.4{ }^{\circ} \mathrm{C}\right)$ and $30 \%\left(\mathrm{O} / \mathrm{N}\right.$ at $\left.4{ }^{\circ} \mathrm{C}\right)$ sucrose in PBS. Then tissues were embedded in OCT compound and sectioned at $14 \mu \mathrm{m}$ (for DRG) or $30 \mu \mathrm{m}$ (for muscle and spinal cord)

For immunohistochemistry, the sections were air dried for $1 \mathrm{~h}$ at room temperature (RT). Antigen retrieval was applied for postnatal DRG and spinal cord staining by immersing the slides in pre-heated $1 \mathrm{x}$ target retrieval solution (Dako, cat\#S1699) for $30 \mathrm{~min}$. The sections were then incubated in blocking solution ( $2 \%$ donkey serum (Jackson ImmunoResearch, cat\#017-000-121), 0.5\% triton and $0.0125 \%$ sodium azide) for $20 \mathrm{~min}$ at RT before applying primary antibodies for $2 \mathrm{O} / \mathrm{N}$ at $4{ }^{\circ} \mathrm{C}$. The primary antibodies used were described in the key resources table. Secondary antibodies Alexa-405, $-488,-555,-647$ (Life Technologies) were applied at 1:250 for $\mathrm{O} / \mathrm{N}$ at $4{ }^{\circ} \mathrm{C}$. DAPI staining was performed together with the secondary antibodies. Sections were then washed 3 times with PBS and mounted with fluorescent mounting medium (Dako, cat\#S3023) for imaging.

For in situ hybridization (RNAscope), the manufacturer's protocol was followed. The probes were designed by the manufacture and available from Advanced Cell Diagnostics. The following probes were used in this study: MmPvalb-C2 (\#421931-C2), Mm-Whrn-C4 (\#511461-C4), Mm-Runx3-C3 (\#451271C3), Mm-Fxyd7-C1 (\#431141), Mm-Tox-C1 (\#484781), Mm-Lmcd1-C1 (\#484761), Mm-Calb1-C2 (\#428431-C2), Mm-Chad-C1 (\#484881), Mm-Aldh1a3C1 (\#501201), Mm-Chodl-C3 (\#450211-C3), Mm-Lmcd1-C2 (\#484761-C2), MmFxyd7-C3 (\#501021-C3), Mm-Calb2-C2 (\#313641-C2), Mm-Tnfaip813-C1 (\#484541), Mm-Vstm2b-C1 (\#484861), Mm-Doc2b-C1 (\#484791), Mm-Hpse-C1 (\#412251), Mm-Cpne4-C1 (\#474721), Hs-Calb1-C2 (\#422161-C2), Mm-Slc17a6C1 (\#319171), Mm-Lipg-C1 (\#492521).

Primary antibodies. Rabbit anti-WHRN (from Joriene de Nooij lab), mouse antiISLET1 (DSHB, cat\#39.4D5), chicken anti-RFP (Rockland, cat\#600-901-379S), rabbit anti-VGLUT1 (SYSY, cat\#135303), guinea pig anti-VGLUT1 (SYSY, cat\#135304), rabbit anti-FXYD7 (Sigma-Aldrich, cat\#HPA026916), rabbit antiLMCD1 (Human Protein Atlas, cat\#HPA024059), goat anti-WGA (Vector Laboratories, cat\#AS-2024), mouse anti-BRN3C (Santa Cruz Biotechnology, cat\#sc-81980), goat anti-PV (Swant, cat\#PVG-213), rabbit anti-PV (Swant, cat\#PV27), rabbit anti-CART (from Igor Adameyko lab), rabbit anti-RUNX1 (from Thomas Jessell lab), rabbit anti-CALB1 (Swant, cat\#CB-38a), goat antiCHAT (Millipore, cat\#AB144p), DAPI (Invitrogen, cat\#D1306)

Image acquisition and analysis. Images were acquired using Zeiss confocal microscope LSM700, LSM800, LSM880, and LSM800 airy equipped with 5x, 10x, 20x, and 40x objectives.

Measurement of soma sizes of PN subtypes. The soma areas of PNs were outlined using freehand selections tool in Fiji and only cells with clear nucleus with DAPI staining were considered. RNAscope was performed for canonical PN markers Pvalb/Runx3 and subtype markers Lmcd1, Chad, Fxyd7, Tox, Aldh1a3, and Chodl to label Ia-, Ib-, II-, $\mathrm{II}_{2^{-}}, \mathrm{II}_{3^{-}}$, and $\mathrm{II}_{4}$-PNs, respectively. Cell bodies were outlined as visualized by Pvalb expression, which fill in the entire cytoplasm and nucleus. Immunostaining was performed for PNs marker PVALB and subtype markers RUNX1, CALB1, and CARTPT to label $\mathrm{Ia}_{1}, \mathrm{Ia}_{2 / 3}$, and $\mathrm{II}_{1}$, respectively, and cell bodies were outlined as visualized by PVALB expression.

Quantification of PN subtypes in Fig. 3d, j, Fig. 5j, and Fig. 6c-f. In Fig. 3d, the proportion of Ia-PNs is quantified from $\mathrm{PNs}\left(\mathrm{PV}^{+}\right)$expressing subtype marker RUNX1 ( $\mathrm{Ia}_{1}-\mathrm{PNs}$ ) and CALB1 ( $\left.\mathrm{Ia}_{2 / 3}-\mathrm{PNs}\right)$ using immunostaining ( $n=3$ animals). Though Runxl is statistically enriched in Ia $\mathrm{a}_{1}$-PNs, Runxl RNA is also lowly expressed in other PN subtypes. However, we observed that RUNX1 as protein is detected in a specific population of PNs with distinct soma size and spatial distribution, which highly suggest that it labels predominantly $\mathrm{Ia}_{1}-\mathrm{PNs}$. The proportion of $\mathrm{Ia}_{3}-\mathrm{PNs}$ is quantified from PNs $\left(\mathrm{Whrn}^{+}\right)$labeled with RFP using RNAscope in $\mathrm{P} 30 \mathrm{Calb2}{ }^{\mathrm{Cre}}$; $\mathrm{Ail} 14$ mice. The proportion of $\mathrm{Ia}_{2}-\mathrm{PNs}$ is the deduction of $\mathrm{Ia}_{3}-\mathrm{PNs}$ from $\mathrm{Ia}_{2 / 3}$-PNs. In Fig. 3j, the proportion of II-PN subtypes is quantified from PNs $\left(\mathrm{Pvalb}^{+} / \mathrm{Run} 3^{+}\right)$expressing subtype markers Tox $\left(\mathrm{II}_{2}-\mathrm{PNs}\right)$, Aldhla3 (II ${ }_{3}$-PNs) and Chodl (II $-\mathrm{PNs}$ ) using RNAscope ( $n=3$ animals). The proportion of
$\mathrm{II}_{1}-\mathrm{PNs}$ is the deduction of $\mathrm{II}_{2^{-}}, \mathrm{II}_{3^{-}}$, and $\mathrm{II}_{4}-\mathrm{PNs}$ from II-PNs. In Fig. $5 \mathrm{~g}, \mathrm{j}$, the proportion of $V s t m 2 b^{+}$and $D o c 2 b^{+}$PNs is quantified from PNs $\left(\right.$Pvalb $^{+} /$Run $\left.3^{+}\right)$ expressing high level of Vstm $2 b$ and $\operatorname{Doc} 2 b$ to represent the distribution of Cl.1 and Cl.3, respectively, since Vstm $2 b$ and Doc $2 b$ is also lowly expressed by cells from other clusters. In Fig. 6c-f, in total, 4-6 sections representing each DRG were used per animal for each immuostaining or RNAscope experiment.

Box and whisker plot. The lower and upper hinges represent the first and third quartiles, respectively, so the box spans the inter-quartile range (IOR). The horizontal line inside the box corresponds to median. The upper whiskers extend to the largest value no further than $1.5 *$ IQR from the upper hinge, while the lower whiskers extend to the smallest value at most $1.5 *$ IQR from the lower hinge. Large dots represent the outliers beyond the range of whiskers.

Free wheel running experiment. Eight-week-old C57BL/6J male mice were singlehoused and randomly assigned into two groups: sedentary (sed) and free wheel running (run). Mice assigned to the free wheel running group were given access to running wheels (Med Associates Inc, cat\#ENV-047) with a counter that monitored revolutions during the experimental period (all animals ran at least $3 \mathrm{~km} /$ day). Mice assigned to the sedentary group were housed in similar cages with locked running wheels for the same period. At the end of the experimental period, the running wheels were removed and animals were sacrificed about $24-48 \mathrm{~h}$ later for tissues collection.

Statistics and reproducibility. Statistical data analysis was performed with Microsoft Excel, presented as mean \pm standard error of mean and statistical significance was calculated using student $t$-tests. Degree of significance was represented as following: ${ }^{*} p \leq 0.05, * * p \leq 0.01, * * * p \leq 0.001, * * * * p \leq 0.0001$. No animals or data points were excluded from the analysis. Our sample sizes are similar to those generally employed in the field. For scRNAseq experiments, replicates were done for all stages: E16.5: 3 plates; P5: 2 plates; P54: 4 plates. For immunostaining and RNAscope experiments, replicates were performed in often $n \geq 3$ animals even in cases where only representative images were presented in the figure.

Single-cell isolation for scRNAseq. The same dissociation protocol was used for DRG of E16.5, P5, and P54 PV Cre; Ailt mice and 3 months old ChAT ${ }^{\mathrm{Cre}} ;$ RGT mice after rabies virus infection. At each stage, DRG were dissected, cut into halves (for postnatal DRGs), and collected in L-15 medium (Life Technologies, cat\#21083027) on ice. The collated DRG were incubated in 2 ml DMEM-F12 medium (Life Technologies, cat\#11039-021) containing $12.5 \mathrm{mg}$ collagenase IV (Life Technologies, cat\#17104019) and $0.8 \mathrm{U}$ dispase $\left(30 \mathrm{~min}\right.$ at $\left.37^{\circ} \mathrm{C}\right)$ then transferred into $1 \mathrm{ml}$ $0.05 \%$ trypsin solution $\left(15 \mathrm{~min}\right.$ at $37^{\circ} \mathrm{C}$ ) (Life Technologies, cat\#25300054), and 1 $\mathrm{ml}$ fetal bovine serum (Life Technologies, cat\#10082147) was added at the end to stop the enzymatic reaction. The tissue was then spun down at $400 \times g$ for $5 \mathrm{~min}$ and resuspended in $1 \mathrm{ml} \mathrm{L}-15$ medium, followed by mechanical trituration using fire polished Pasteur pipettes until the solution homogenized and filtered through $70 \mu \mathrm{m}$ cell strainer (BD Biosciences, cat $\# 352350$ ). Single $\mathrm{RFP}^{+}$cells were sorted by fluorescence-activated cell sorting (FACS) into individual wells containing lysis buffer in a 384-well plate. The plates were immediately placed on dry ice and stored at $-80^{\circ} \mathrm{C}$ before processed for Smart-seq 2 protocol. For the rabies virus traced cells, single $\mathrm{GFP}^{+}$cells were picked with a robotic cell-picking setup, constructed in-house around an inverted Nikon microscope. Individual cells were sucked into a glass capillary attached to a CellTram syringe with visual control. By an automated $x-y-z$ axis drive, the capillary tip was positioned in a well of a 48-well plate containing $3 \mu \mathrm{l}$ of lysis buffer, into which the cell was released. After cell picking, the plates were immediately placed on dry ice and stored at $-80^{\circ} \mathrm{C}$ before further procedures.

Single-cell RNA-sequencing. Smart-Seq2 protocol was performed on single isolated cells in the lab (for the rabies virus traced PNs) or by Eukaryotic Single Cell Genomics Facility at SciLifeLab, Stockholm (for the E16.5, P5 and P54 PNs). The protocol was described previously ${ }^{12,51}$.

scRNAseq data analysis. The data pre-processing (demultiplexing, annotation) was done by Eukaryotic Single Cell Genomics Facility at SciLifeLab, Stockholm using validated methods. Most of the downstream analysis of E16.5, P5, and P54 data followed the same pipeline using R package Seurat ${ }^{13}$. A step-to-step analysis of adult data is described below. The detailed analysis of E16.5 and P5 data is not be repeated in the "Methods" section, except for the selection of key parameters. The codes used in this study will be released through the lab Github channel.

Filtration and normalization. We obtained in total four 384-well plates for P54 PNs from two individual preparations (preparation 1: plate 1 and 2, preparation 2: plate 3 and 4). Subtle technical variations were observed between preparations but not plates from the same preparation. We thus created two Seurat objects representing two preparations and processed with the same filter and normalization procedures. 
To remove empty wells and low-quality cells, we selected cells with at least 7500 unique genes detected for downstream analysis. Genes expressed in less than three cells were also removed from analysis. To be able to integrate the two preparations, we used a global normalization strategy incorporated in Seurat package to make the data comparable between different cells and between different Smart-seq2 runs. The gene expression measurements within each cell were scaled by a constant factor 100,000, then natural-log transformed. This generated a new gene expression matrix $y_{\mathrm{i}, \mathrm{j}}=\log \left(x_{\mathrm{i}, \mathrm{j}} Z_{\mathrm{j}} * 100,000\right)$, where $x_{\mathrm{i}, \mathrm{j}}$ is the count of gene $i$ in cell $j$ and $Z_{\mathrm{i}, \mathrm{j}}$ is the total counts of all genes in cell $j$.

We calculated the dispersion (ratio of variation to mean) of genes and selected those highly variable genes from each preparation for the alignment of the two preparations. We also regressed out the cell-cell variation in gene expression driven by "percent.mito (the percentage of mitochondrial gene content)" using the ScaleData function.

Data integration. To reveal the shared sources of biological variation between the two preparations, we used the integration tool for scRNAseq data sets provided by Seurat package. This tool has shown better performance over other benchmarking alignment and batch correction tools, e.g., limma, ComBat. It comprises Canonical Correlation Analysis (CCA), which is a method of inferring relationship from two matrices. Given two groups of cells $X=(\mathrm{X} 1, \mathrm{X} 2, \ldots, \mathrm{X} m)$ and $Y=(\mathrm{Y} 1, \mathrm{Y} 2, \ldots, \mathrm{Y} n)$, and if there were correlations among them, the analysis would determine a set of canonical variates (CVs, referred to as CCs in Seurat package), storing linear combinations of $X_{\mathrm{i}}$ and $Y_{\mathrm{j}}$, which have maximal correlation with each other. Using a union of most variable genes from both preparations as the input for CCA, we selected the first 18 CCs to approximate all CCs, which project both data sets into the maximally correlated subspace.

Clustering and dimensional reduction. In order to obtain an overview of heterogeneity in the integrated P54 data, we ran the function RunTSNE using the first 18 CCs to visualize cells in the two-dimensional space and used the function FindClusters (resolution $=0.8$ ) to identify clusters of cells. This resulted in ten clusters, among which three clusters were identified to be glia-contaminated PNs $\left(\right.$ Sox $\left.10^{+}\right), \operatorname{Ret}^{+}$, and $\mathrm{Ntrk}^{+}$mechanoreceptors, respectively, and removed from the following analysis. The unbiased clustering analysis revealed seven clusters of molecularly distinct PNs, among which we observed that one cluster (later split into $\mathrm{Cl} .2$ and $\mathrm{Cl} .3$ ) exhibited large heterogeneity in the gene expression. Cl.3, identifiable by its specific expression of Calb2, also had enriched/specific expression of many other genes (Supplementary Fig. 1e and Supplementary Dataset 2). With the notion to not overlook the diversity of PNs, we listed Cl.3 as an individual cluster, which we believe could be revealed by unbiased clustering analysis through sequencing more PNs. These eight clusters were then clustered into three major clusters based on similarity of expression of highly specific markers unbiasedly identified, and later confirmed by their expression in situ and through analysis of their innervation profile.

Differential expression analysis. To identify differentially expressed genes in each cluster, we used "MAST" test implemented in the function FindMarkers ${ }^{52}$. The parameters "min.pct $=0.25$ " and "logfc.threshold $=0.3$ " are chosen to select genes expressed in more than $25 \%$ of cells and at least 0.3 -fold difference (log-scale) in the given cluster. From the list of differentially expressed genes, all the genetic markers used in this study were further shortlisted based on their expression level and high specificity in the given cluster. As example, the expression of adult Ib-PNs marker Chad is 2.7-fold (log-scale) higher in Ib-PNs compared with other PNs. Also, we have used a stringent cutoff for a gene expression to be considered positive and to be shown or discussed in the text, considering only the $50 \%$ most expressed genes from our dataset to be represented and discussed.

Projection of scRNAseq data across data sets. To unbiasedly assign virus-traced cells to P54 PNs clusters, we used the R package scmap ${ }^{21}$ to project the scRNAseq data set of the virus-traced cells to the reference data set, the P54 scRNAseq data set. For this, similarities between each cell and all centroids of the reference data set are calculated using three different measurement: Pearson, Spearman, and cosine. The cell is then assigned to the cell type that corresponds to the highest similarity value. Only when at least two similarities agree with each other and at least one similarity score is above 0.7, the cell could be assigned. P5 PNs were assigned to major types of adult PNs using the same method.

Selection of key parameters for E16.5 and P5 data analysis. At E16.5, different PN types are establishing connections with their respective central and peripheral targets, so we reasoned that major PN types should be distinguishable at transcriptomic level at this stage, as recently suggested in a study by the lab of Joriene de Nooij ${ }^{41}$. For the initial clustering of E16.5 data, a low resolution of 0.3 was chosen and identified 3 major clusters (Fig. 5a). Since heterogeneity was observed within the major clusters, we increased the resolution to 0.8 , resulting in seven clusters, which appeared to be in agreement with the structure of the tSNE plot (Supplementary Fig. 5b). For the analysis of P5 data, a resolution of 0.8 was used. Owing to small number of cells, cluster6 at P5 was selected manually based on its high specific Chodl expression.
Reporting summary. Further information on research design is available in the Nature Research Reporting Summary linked to this article.

\section{Data availability}

All data is available in the main text or the supplementary materials, apart from the scRNAseq transcriptomic data, which is accessible at GEO data repository (accession code: GSE156180). A browsable resource of E16.5, P5, and P54 PNs data is available at the lab website: https://ki.se/en/neuro/lallemend-laboratory. Source data are provided with this paper.

Received: 3 August 2020; Accepted: 15 January 2021; Published online: 15 February 2021

\section{References}

1. Sherrington, C. S. Strychnine and reflex inhibition of skeletal muscle. J. Physiol. 36, 185-204 (1907).

2. Takeoka, A., Vollenweider, I., Courtine, G. \& Arber, S. Muscle spindle feedback directs locomotor recovery and circuit reorganization after spinal cord injury. Cell 159, 1626-1639 (2014).

3. Matthews, P. B. Muscle spindles and their motor control. Physiol. Rev. 44, 219-288 (1964).

4. Granit, R. The functional role of the muscle spindles-facts and hypotheses. Brain 98, 531-556 (1975).

5. Sherrington, C. S. Flexion-reflex of the limb, crossed extension-reflex, and reflex stepping and standing. J. Physiol. 40, 28-121 (1910).

6. Kiehn, O. Decoding the organization of spinal circuits that control locomotion. Nat. Rev. Neurosci. 17, 224-238 (2016).

7. Sathyamurthy, A. et al. Massively parallel single nucleus transcriptional profiling defines spinal cord neurons and their activity during behavior. Cell Rep. 22, 2216-2225 (2018).

8. Sharma, N. et al. The emergence of transcriptional identity in somatosensory neurons. Nature 577, 392-398 (2020).

9. Zeisel, A. et al. Molecular architecture of the mouse nervous system. Cell 174, 999-1014 e1022 (2018)

10. Li, C. L. et al. Somatosensory neuron types identified by high-coverage singlecell RNA-sequencing and functional heterogeneity. Cell Res. 26, 967 (2016).

11. Usoskin, D. et al. Unbiased classification of sensory neuron types by largescale single-cell RNA sequencing. Nat. Neurosci. 18, 145-153 (2015).

12. Picelli, S. et al. Smart-seq2 for sensitive full-length transcriptome profiling in single cells. Nat. Methods 10, 1096-1098 (2013).

13. Butler, A., Hoffman, P., Smibert, P., Papalexi, E. \& Satija, R. Integrating singlecell transcriptomic data across different conditions, technologies, and species. Nat. Biotechnol. 36, 411-420 (2018).

14. Gasser, H. S. \& Grundfest, H. Axon diameters in relation to the spike dimensions and the conduction velocity in mammalian A fibers. Am. J. Physiol.-Leg. Content 127, 393-414 (1939).

15. Hursh, J. B. Conduction velocity and diameter of nerve fibers. Am. J. Physiol.Leg. Content 127, 131-139 (1939).

16. Ruffini, A. On the minute anatomy of the neuromuscular spindles of the cat, and on their physiological significance. J. Physiol. 23, 190-208 193 (1898).

17. Cote, M. P., Murray, L. M. \& Knikou, M. Spinal control of locomotion: individual neurons, their circuits and functions. Front. Physiol. 9, 784 (2018).

18. Edgley, S. A. \& Jankowska, E. An interneuronal relay for group I and II muscle afferents in the midlumbar segments of the cat spinal cord. J. Physiol. 389, 647-674 (1987).

19. de Nooij, J. C., Doobar, S. \& Jessell, T. M. Etv1 inactivation reveals proprioceptor subclasses that reflect the level of NT3 expression in muscle targets. Neuron 77, 1055-1068 (2013).

20. Takatoh, J. et al. New modules are added to vibrissal premotor circuitry with the emergence of exploratory whisking. Neuron 77, 346-360 (2013).

21. Kiselev, V. Y., Yiu, A. \& Hemberg, M. scmap: projection of single-cell RNAseq data across data sets. Nat. Methods 15, 359-362 (2018).

22. Critchlow, V. \& Von, E. Intercostal muscle spindle activity and its gamma motor control. J. Physiol. 168, 820-847 (1963).

23. Woo, S. H. et al. Piezo2 is the principal mechanotransduction channel for proprioception. Nat. Neurosci. 18, 1756-1762 (2015).

24. Adams, J. C. Cell-matrix contact structures. Cell Mol. Life Sci. 58, 371-392 (2001).

25. Camper, L., Heinegard, D. \& Lundgren-Akerlund, E. Integrin alpha2beta1 is a receptor for the cartilage matrix protein chondroadherin. J. Cell Biol. 138, 1159-1167 (1997).

26. Zheng, Y. et al. Deep sequencing of somatosensory neurons reveals molecular determinants of intrinsic physiological properties. Neuron 103, 598-616 e597 (2019). 
27. Branco, T. \& Staras, K. The probability of neurotransmitter release: variability and feedback control at single synapses. Nat. Rev. Neurosci. 10, 373-383 (2009).

28. Zimmerman, A. L. et al. Distinct modes of presynaptic inhibition of cutaneous afferents and their functions in behavior. Neuron 102, 420-434 e428 (2019).

29. Koch, S. C., Acton, D. \& Goulding, M. Spinal circuits for touch, pain, and itch. Annu. Rev. Physiol. 80, 189-217 (2018).

30. Sando, R. 3rd et al. Inducible control of gene expression with destabilized Cre. Nat. Methods 10, 1085-1088 (2013).

31. Özel, M. N. et al. Neuronal diversity and convergence in a visual system developmental atlas. Nature 589, 88-95 (2021).

32. Flavell, S. W. \& Greenberg, M. E. Signaling mechanisms linking neuronal activity to gene expression and plasticity of the nervous system. Annu. Rev. Neurosci. 31, 563-590 (2008).

33. Hensch, T. K. Critical period plasticity in local cortical circuits. Nat. Rev. Neurosci. 6, 877-888 (2005).

34. Dayan, E. \& Cohen, L. G. Neuroplasticity subserving motor skill learning. Neuron 72, 443-454 (2011).

35. Statton, M. A., Encarnacion, M., Celnik, P. \& Bastian, A. J. A single bout of moderate aerobic exercise improves motor skill acquisition. PLoS ONE 10, e0141393 (2015).

36. Li, H. Q. \& Spitzer, N. C. Exercise enhances motor skill learning by neurotransmitter switching in the adult midbrain. Nat. Commun. 11, 2195 (2020).

37. Adkins, D. L., Boychuk, J., Remple, M. S. \& Kleim, J. A. Motor training induces experience-specific patterns of plasticity across motor cortex and spinal cord. J. Appl. Physiol. 101, 1776-1782 (2006). (1985).

38. Klintsova, A. Y., Dickson, E., Yoshida, R. \& Greenough, W. T. Altered expression of BDNF and its high-affinity receptor TrkB in response to complex motor learning and moderate exercise. Brain Res. 1028, 92-104 (2004).

39. Lallemend, F. \& Ernfors, P. Molecular interactions underlying the specification of sensory neurons. Trends Neurosci. 35, 373-381 (2012).

40. Wang, Y. et al. A cell fitness selection model for neuronal survival during development. Nat. Commun. 10, 4137 (2019).

41. $\mathrm{Wu}, \mathrm{D}$. et al. A role for sensory end organ-derived signals in regulating muscle spindle proprioceptor phenotype. J. Neurosci. 39, 4252-4267 (2019).

42. De Marco Garcia, N. V., Karayannis, T. \& Fishell, G. Neuronal activity is required for the development of specific cortical interneuron subtypes. Nature 472, 351-355 (2011)

43. Spitzer, N. C. Electrical activity in early neuronal development. Nature 444, 707-712 (2006).

44. Hippenmeyer, S. et al. A developmental switch in the response of DRG neurons to ETS transcription factor signaling. PLoS Biol. 3, e159 (2005).

45. Madisen, L. et al. A robust and high-throughput Cre reporting and characterization system for the whole mouse brain. Nat. Neurosci. 13, 133-140 (2010).

46. Rossi, J. et al. Melanocortin-4 receptors expressed by cholinergic neurons regulate energy balance and glucose homeostasis. Cell Metab. 13, 195-204 (2011).

47. Taniguchi, H. et al. A resource of Cre driver lines for genetic targeting of GABAergic neurons in cerebral cortex. Neuron 71, 995-1013 (2011).

48. Daigle, T. L. et al. A suite of transgenic driver and reporter mouse lines with enhanced brain-cell-type targeting and functionality. Cell 174, 465-480 e422 (2018).

49. Quadros, R. M. et al. Easi-CRISPR: a robust method for one-step generation of mice carrying conditional and insertion alleles using long ssDNA donors and CRISPR ribonucleoproteins. Genome Biol. 18, 92 (2017).

50. Miura, H., Quadros, R. M., Gurumurthy, C. B. \& Ohtsuka, M. Easi-CRISPR for creating knock-in and conditional knockout mouse models using long ssDNA donors. Nat. Protoc. 13, 195-215 (2018).

51. Picelli, S. et al. Full-length RNA-seq from single cells using Smart-seq2. Nat. Protoc. 9, 171-181 (2014).
52. Finak, G. et al. MAST: a flexible statistical framework for assessing transcriptional changes and characterizing heterogeneity in single-cell RNA sequencing data. Genome Biol. 16, 278 (2015).

\section{Acknowledgements}

We thank Prof. Fan Wang and Dr. Joriene de Nooij for the RGT and Egr $3^{W G A}$ mouse lines, respectively, the CLICK imaging Facility supported by the Knut and Alice Wallenberg Foundation, the Uppsala Multidisciplinary Center for Advanced Computational Science (UPPMAX) for providing data storage resources, and Kuisong Song for technical implication in some results of Fig. 5j. We are also grateful to Prof. Sten Grillner for critical reading of the manuscript. This work was supported by grants from: StratNeuro, the Swedish Brain Foundation, Karolinska Institutet, the Swedish Research Council, KID funding (F.L. and S.H.); the Knut and Alice Wallenbergs Foundation (Wallenberg Academy Fellow), Ragnar Söderberg Foundation (Ragnar Söderberg Fellow in Medicine) and Ming Wai Lau Foundation (F.L.). F.L. is a Wallenberg Academy Fellow in Medicine and a MWLC investigator.

\section{Author contributions}

F.L. conceived and supervised the project, with inputs from H.W. and S.H. H.W. and F.L designed experiments; H.W., C.P., P.F., A.S., C.B., Y.W., K.K.Y.C., S.W., and P.J. performed experiments and analyzed data; R.M.Q., J.A.H., Y.X., K.M., J.R., C.B.G., and O.K. provided unique reagents and mouse models. H.W, S.H., and F.L. wrote the manuscript with inputs from all co-authors.

\section{Funding}

Open Access funding provided by Karolinska Institute.

\section{Competing interests}

The authors declare no competing interests.

\section{Additional information}

Supplementary information The online version contains supplementary material available at https://doi.org/10.1038/s41467-021-21173-9.

Correspondence and requests for materials should be addressed to F.L.

Peer review information Nature Communications thanks Matthew Kelley and the other anonymous reviewer(s) for their contribution to the peer review of this work. Peer reviewer reports are available.

Reprints and permission information is available at http://www.nature.com/reprints

Publisher's note Springer Nature remains neutral with regard to jurisdictional claims in published maps and institutional affiliations.

Open Access This article is licensed under a Creative Common Attribution 4.0 International License, which permits use, sharing, adaptation, distribution and reproduction in any medium or format, as long as you give appropriate credit to the original author(s) and the source, provide a link to the Creative Commons license, and indicate if changes were made. The images or other third party material in this article are included in the article's Creative Commons license, unless indicated otherwise in a credit line to the material. If material is not included in the article's Creative Commons license and your intended use is not permitted by statutory regulation or exceeds the permitted use, you will need to obtain permission directly from the copyright holder. To view a copy of this license, visit http://creativecommons.org/ licenses/by/4.0/.

(C) The Author(s) 2021 